\title{
Interpretation of Dark Matter and Quark-Gluon Plasma: The Generation of the Periodic Table Elements and Its Phase Diagram: A Novel Millennium Power Plant
}

\author{
Murad Al Shibli \\ Autonomous Systems Engineering Technology Department, Head, Senior Faculty, Abu Dhabi Polytechnic, \\ Institute of Applied Technology, Abu Dhabi, UAE \\ Email: murad.alshibli@iat.ac.ae
}

Received 20 June 2015; accepted 20 September 2015; published 23 September 2015

Copyright (C) 2015 by author and Scientific Research Publishing Inc.

This work is licensed under the Creative Commons Attribution International License (CC BY). http://creativecommons.org/licenses/by/4.0/

(c) (i) Open Access

\section{Abstract}

This paper presents a novel physical interpretation of the state of matter of the quark-gluon as the most fundamental building blocks in nature. Such a model is derived based on the assumption that dark matter and dark energy behave as a perfect ideal fluid at extremely high temperature. By the virtue of Boltzmann constant of the ideal gas law and NASA's Cosmic Microwave Background Explorer (CMB) which estimate that the space has an average temperature close to 2.7251 Kelvin, then the equivalent mass-energy of the fundamental particle of the dark matter/dark energy is determined. Moreover, assuming a uniform space dark energy/dark matter density, then the critical temperature at which the dark matter has a unity entity per volume is identified as $64 \times 10^{12} \mathrm{~K}$. The calculated critical temperature of the quark-gluon plasma is found to be proportional to the temperature generated by colliding heavy ions at the Relativistic Heavy Ion Collider (RHIC) and European Organization for Nuclear Research (CERN). Moreover, the individual critical temperatures of the quark-gluon plasma matter at which the elements of the Periodic Table are generated are explicitly determined. The generation temperature trend of the elements of the Periodic Table groups and Periods is then demonstrated. Accordingly, the phase diagram of the quark-gluon state matter is proposed. Finally, a new model of quark-gluon power generation plant is proposed and aims to serve humanity with new energy sources in the new millennium.

\section{Keywords}

Dark Matter, Dark Energy, Quark-Gluon Plasma, Equation of State, Periodic Table, Standard Model 


\section{Introduction}

Recent astronomical observations by the cosmic microwave background (CMB), Supernova Cosmology Project, and High-z Supernova Search Team have provided strong evidence that our universe is not only expanding, but also expanding at an accelerating rate [1]-[8]. Dark Energy was proposed for the first time in 1998 when two groups of astronomers made a survey of exploding stars, or supernovas Ia, in a number of distant galaxies [1] [3]. Those researchers found that the supernovas were dimmer than they should have been and were farther away than they should have been. The astronomers realized the only way for that to happen if the expansion of the universe had sped up at some time in the past which should account for a significant portion of a missing component in the universe [9]. The only explanation was that there was a kind of force that has a strong negative pressure acting outward in opposition to gravitational force at large scales which was proposed for the first time by Einstein in his General Relativity and given the name the cosmological constant Lambda [10]. This force is given the name Dark Energy since it is transparent and cannot be observed or detected directly. A thermodynamics model is proposed to account for the dark energy in [11]. Cosmological observations strongly suggest that the universe is dominated by a smoothly homogenous distributed dark energy component [12]-[21]. It was worth mentioning that NASA's Cosmic Microwave Background Explorer (CMB) in 1992 estimated that the sky had a temperature close to 2.7251 Kelvin. Moreover, the Wilkinson Microwave Anisotropy Probe (WMAP) in 2003 has made a map of the temperature fluctuations of the CMB with more accuracy [12]. Furthermore, the measurements of the Wilkinson Microwave Anisotropy Probe (WMAP) satellite indicate that the universe has a flat geometry [22]-[25].

Using the Doppler Shift phenomena, scientists can learn much about the motions of galaxies. When scientists look closer at the speeds of galactic rotation, they find something strange. Based on Keplerian physics, the individual stars in a galaxy should act like the planets in our solar system - the farther away from the center, the slower they should move. On the contrary, the Doppler Shift reveals that the stars in many galaxies do not slow down at farther distances [1] [12]. Particularly, observations reveal that the stars move at flat speeds that should rip the galaxy apart because there is not enough measured mass to supply the gravity needed to hold the galaxy together. These high rotational speeds suggest that the galaxy contains more mass than was calculated. Scientists theorized that, if the galaxy was surrounded by a halo of unseen matter, the galaxy could remain stable at such high rotational speeds. Accordingly, dark matter can be defined as the unseen matter of unknown composition that does not emit or reflect enough electromagnetic radiation to be observed directly, but its presence can be inferred from gravitational effects on visible matter like galaxies and stars. Based on the Lambda-Cold Dark Matter Model (Lambda-CDM 2006), dark energy contributes about 70\% of the critical density and has a negative pressure [23] [24]. The cold dark matter contributes 25\% [1]-[3] [12] [19] [20] [25] [26]. Meanwhile, hydrogen, helium and stars contribute $5 \%$ and, finally the radiation contributes $5 \times 10^{-5}$. According to present observations of structures larger than galaxy-sized as well as Big Bang cosmology, dark matter accounts for the vast majority of mass in the observable universe $(22 \%)$. The observed phenomena consistent with dark matter observations include the rotational speeds of galaxies, orbital velocities of galaxies in clusters, gravitational lensing of background objects by galaxy clusters, and the temperature distribution of hot gas in galaxies and clusters of galaxies.

A quark-gluon plasma (QGP) can be defined as a phase of quantum chromodynamics (QCD) which exists at extremely high temperature and/or density. This phase consists of (almost) free quarks and gluons, which are several of the basic building blocks of matter. Recent analyses from the Relativistic Heavy Ion Collider (RHIC), a 2.4-mile-circumference (atom smasher) at the U.S. Department of Energy's (DOE) Brookhaven National Laboratory, establish that collisions of gold ions traveling at nearly the speed of light have created matter at a temperature of about 4 trillion degrees Celsius - the hottest temperature ever reached in a laboratory, about 250,000 times hotter than the center of the Sun [27]-[31]. This temperature, based upon measurements by the PHENIX collaboration at RHIC, is higher than the temperature needed to melt protons and neutrons into plasma of quarks and gluons. These new temperature measurements, combined with other observations analyzed over nine years of operations by RHIC's four experimental collaborations-BRAHMS, PHENIX, PHOBOS, and STAR-indicate that RHIC's gold-gold collisions produce a freely flowing liquid composed of quarks and gluons. Such a substance, often referred to as quark-gluon plasma, or QGP, filled the universe a few microseconds after it came into existence 13.7 billion years ago.

RHIC has created a new state of hot, dense matter out of the quarks and gluons that are the basic particles of 
atomic nuclei, but it is a state quite different and even more remarkable than had been predicted. When nuclear matter is heated beyond 2 trillion degrees, it becomes strongly coupled plasma of quarks and gluons. Experiments using highly energetic collisions between heavy nuclei have revealed that this new state of matter is a nearly ideal, highly opaque fluid [32].

The expansion of hot and dense matter created in a heavy ion collision at RHIC is controlled by an equation of state which describes the dependence of the pressure in the medium on its energy density. Knowledge of the equation of state is, for instance, indispensable for a correct hydrodynamic modeling of the expansion of the transient form of matter created in a gold-gold collision at RHIC [33]. The new results on the equation of state show features familiar from earlier approximate calculations; a rapid rise of the energy density in the transition region at about $1 \mathrm{GeV} / \mathrm{fm}^{3}$ or a temperature of about $190 \mathrm{MeV}$ and a comparatively slow increase of the pressure which comes close to that of a quasi-free quark-gluon gas only at temperatures of about $600 \mathrm{MeV}$. In fact, even at these high temperatures it stays about $10 \%$ below the ideal gas limit, which is known to be reached eventually at extremely high temperatures. High statistics results on large lattices are obtained for bulk thermodynamic observables, i.e. pressure, energy and entropy density, at vanishing quark chemical potential for a wide range of temperatures, $140 \mathrm{MeV} \leq \mathrm{T} \leq 800 \mathrm{MeV}$.

This paper introduces a novel physical interpretation of the state of matter of the quark-gluon as the most fundamental building blocks in nature. Such a model is derived based on the assumption that dark matter and dark energy behave as a perfect ideal fluid at extremely high temperature as presented in Section 2. By the virtue of Boltzmann constant of the ideal gas law and NASA's Cosmic Microwave Background Explorer (CMB) which estimated that the space has an average temperature close to 2.7251 Kelvin, then the equivalent massenergy of the fundamental particle of the dark matter/dark energy is determined. The calculated critical temperature of the quark-gluon plasma is found to be proportional to the temperature generated by colliding heavy ions at the Relativistic Heavy Ion Collider (RHIC) and European Organization for Nuclear Research (CERN). Moreover, assuming a uniform space dark energy/dark matter density, then the critical temperature at which the dark matter has a unity entity per volume is identified as $64 \times 10^{12} \mathrm{~K}$ as derived in Section 3 . The quark-gluon standard model of the proton and Neutron is presented in Section 4. Then, an isothermal expansion model of the universe is proposed in Section 5. Moreover, the individual critical temperature of the quark-gluon plasma matter at which the elements of the Periodic Table are generated is explicitly determined in Section 6. The generation temperature trend of the elements of the Periodic table groups and Periods are then demonstrated. Accordingly, a novel phase diagram of the quark-gluon state matter is proposed and a new model of quark-gluon power generation plant is proposed in Section 7 that aims to serve humanity with new energy sources in the new millennium. Finally, conclusions are presented in Section 8.

\section{Preliminary Review on the Basic Blocks of the Universe}

This state of matter equation relates the pressure $P$, temperature $T$ and the volume $V$ of a substance behaves as an ideal gas [34], that is

$$
P V=m R T
$$

As it can be seen easily that Equation (1) represents the energy associated with an ideal gas at given pressure $P$, temperature $T$ and the volume $V$, that is

$$
P V=m R T=E
$$

Note that both sides of the equation has the units of energy (work done by pressure $P$ ). Assume now that dark energy behaves like an ideal gas with a negative pressure $(-P)$ that causes the universe to expand with a total volume $V$, then by dividing both side of the equation of state (5) by $V$, then

$$
P=\frac{m}{V} R T=\frac{E}{V}
$$

Defining the mass density as $\rho_{m}=\frac{m}{V}$ and energy density as $\rho_{E}=\frac{E}{V}$, Equation (3) yields to

$$
P=\rho_{m} R T=\rho_{E}
$$

Now by taking the ratio between the mass density and energy density then 


$$
\frac{\rho_{E}}{\rho_{m}}=R T
$$

It can be concluded that the ratio between the mass density and energy density are proportional to the product of the temperature $T$ and dark energy-dark matter constant $R$ (known as Universal gas constant).

The Boltzmann constant $K_{B}$ is a physical constant that relates temperature to microscopic energy.

$K_{B}=R / N_{A}$, where $N_{A}$ is the Avogadro Number. $K_{B}=1.38 \times 10^{-23} \mathrm{~J} / \mathrm{K}$. The numerical value of $K_{B}$ measures the conversion factor for mapping from this microscopic energy $E$ to the macroscopically-derived temperature scale.

The ideal gas law can now be expressed in terms of Boltzmann constant such that

$$
P V=N K_{B} T
$$

where $N$ is the actual number of entities (particles). Now dividing both sides of (10) by volume to get the energy density then

$$
P=\frac{N}{V} K_{B} T=\rho_{N} K_{B} T=\rho_{E}
$$

This shows that the ratio between the energy density and the entities density is proportional to the absolute temperature times the Boltzmann constant.

The ideal gas law can now be expressed in terms of Boltzmann constant such that

$$
P V=N k_{B} T=E
$$

where $N$ is the actual number of molecules. Now dividing both sides of (8) by the volume to get the energy density then

$$
P=\frac{N}{V} k_{B} T=\rho_{N} k_{B} T=\rho_{E}
$$

By taking the ration between the energy density $\rho_{E}$ and number of molecules density $N / V$, one gets

$$
k_{B} T=\frac{\rho_{E}}{\rho_{N}}
$$

or

$$
\rho_{N}=\frac{\rho_{E}}{k_{B} T}
$$

This shows that the ratio between the energy density and the molecular density is proportional to the absolute temperature times the Boltzmann constant. The simulation results demonstrate such a model.

\section{Dark Matter, Its Critical Temperature and Physical Properties}

WMAP [8] determined that the universe is flat, from which it follows that the mean energy density in the universe is equal to the critical density (within a $1 \%$ margin of error). This is equivalent to a mass density of $9.9 \times$ $10^{-30} \mathrm{~g} / \mathrm{cm}^{3}$, which is equivalent to only 5.9 protons per cubic meter.

$$
\begin{aligned}
\rho_{\mathrm{E}} & =9.9 \times 10^{-30} \mathrm{gm} / \mathrm{cm}^{3}=9.9 \times 10^{-27} \mathrm{~kg} / \mathrm{m}^{3} \\
& =8.8981 \times 10^{-10} \mathrm{~J} / \mathrm{m}^{3}=5.5302 \times 10^{9} \mathrm{eV} / \mathrm{m}^{3}=5.5302 \mathrm{GeV} / \mathrm{m}^{3}
\end{aligned}
$$

Now the equation of state can be expressed as a function of the number of entities per cubic meter such that,

$$
T=\frac{\rho_{\mathrm{E}}}{\rho_{\mathrm{N}} K_{B}}=6.4478 \times 10^{13} \frac{1}{\rho_{\mathrm{N}}} \mathrm{K}=\frac{64.478}{\rho_{\mathrm{N}}} \mathrm{TK}
$$

The temperature at which one entity would be generated called in this work as the critical temperature and equals to 64 Tetra Kelvin. It is desired now to calculate the critical temperature at which 5.9 protons per cubic meter were produced. By the virtue of the ideal state equation, it yields 


$$
\begin{aligned}
T & =\frac{\rho_{\mathrm{E}}}{\rho_{\mathrm{N}} K_{B}}=\frac{8.8981 \times 10^{-10} \mathrm{~J} / \mathrm{m}^{3}}{5.9 \times 1.38 \times 10^{-23} \mathrm{~J} / \mathrm{K}}=10.929 \times 10^{12} \mathrm{~K} / \mathrm{m}^{3} \\
& =10.929 \text { Tetra } \mathrm{K} / \mathrm{m}^{3}=10.929 \mathrm{TK} / \mathrm{m}^{3}
\end{aligned}
$$

It implies that 10.929 Trillion Kelvin degrees were needed to produce an equivalent 5.9 protons per cubic meter of mass-energy critical density. Furthermore, $\rho_{N}$ is unity when the temperature $T$ is equal to $64.479 \times$ $10^{12} \mathrm{~K}$ (this would be the temperature to generate 1 protons per cubic meter).

Based on astronomical observations that the average density of dark matter and dark energy is approximately:

$$
\begin{aligned}
\rho_{\mathrm{DM}} & =0.23 \times 9.9 \times 10^{-30} \mathrm{gm} / \mathrm{cm}^{3}=2.2770 \times 10^{-27} \mathrm{~kg} / \mathrm{m}^{3} \\
& =2.0466 \times 10^{-10} \mathrm{~J} / \mathrm{m}^{3}=1.2719 \times 10^{9} \mathrm{eV} / \mathrm{m}^{3}=1.2719 \mathrm{GeV} / \mathrm{m}^{3}
\end{aligned}
$$

Now benefiting from (11) at which CMB temperature is $T=2.73 \mathrm{~K}$, then

$$
\rho_{N}=6.4480 \times 10^{12} \text { entities } / \mathrm{m}^{3}
$$

Since $\rho_{N}=6.4480 \times 10^{12}$ entities $/ \mathrm{m}^{3}$ is corresponding to $2.2770 \times 10^{-27} \mathrm{~kg} / \mathrm{m}^{3}$, then each entity has a mass of $2.2770 \times 10^{-27} \mathrm{~kg} / \mathrm{m} / 6.4480 \times 10^{12}=3.5313 \mathrm{e} \times 10^{-40} \mathrm{~kg}$. This may serve as the most fundamental particle in nature and represent the basic block of the quarks and gluons. Such a mass $3.5313 \mathrm{e} \times 10^{-40} \mathrm{~kg}$ is equivalent to $3.1739 \mathrm{e} \times 10^{-23} \mathrm{~J}=1.9726 \times 10^{-4} \mathrm{eV}=0.19726 \mathrm{meV}$. It can be noticed that this particle mass $3.1739 \mathrm{e} \times 10^{-23} \mathrm{~J}$ is proportional to Boltzmann constant $K_{B}=1.38 \times 10^{-23} \mathrm{~J} / \mathrm{K}$.

Moreover, considering the lowest temperature in nature at Boomerang nebula which is 1 Kelvin, then the dark matter should be exactly equivalent to Boltzmann constant, which is $m_{B}=1.38 \times 10^{-23} \mathrm{~J}$.

As it can be seen, the mass of the electron is much heavier than this candidate particle by 2.5796 billion times, meanwhile, the proton weighs 4.7365 trillion $\left(4.7365 \times 10^{12}\right)$ of dark matter particles. The atomic mass unit $\mathrm{atmu}=0.93146 \mathrm{GeV} / \mathrm{c}^{2}$. Now let us compare the mass of the dark matter particle to the standard value of (at$\mathrm{mu}$ ), which leads to the conclusion that 4.7220 Trillion $\left(4.7220 \times 10^{12}\right)$ dark matter particles are required to construct 1 unity of atomic mass unit. In other words, each dark matter particle weighs $\left(2.1177 \times 10^{-13}\right)$ atmu.

It is possible now to calculate the virtual momentum that dark matter particle possesses as follows:

$$
\bar{p}=E / c=1.0587 \times 10^{-31} \mathrm{~kg} \cdot \mathrm{m} / \mathrm{s}
$$

Moreover, the dark matter particle mean life time $\tau$ can be determined using Blank constant $\bar{h}=h / 2 \pi=6.58 \times 10^{-16} \mathrm{eV} \cdot \mathrm{s}$ such that

$$
\tau=\frac{\bar{h}}{E}=\frac{6.58 \times 10^{-16} \mathrm{eV} \cdot \mathrm{s}}{1.9726 \times 10^{-4} \mathrm{eV}}=3.3357 \times 10^{-12} \mathrm{~s}=3.3357 \mathrm{ps}
$$

Additionally, based on the mean life time $\tau$, then one can calculate the frequency of dark matter

$$
f=1 / \tau=0.29979 \times 10^{12} \mathrm{~Hz}=0.29979 \mathrm{THz}
$$

Finally the virtual wave length of such dark matter particle would be

$$
\lambda=\tau \cdot c=0.001 \mathrm{~m}=1 \mathrm{~mm}
$$

It can be concluded that it takes the dark matter particle $3.3357 \mathrm{ps}$ to travel $1 \mathrm{~mm}$. Meanwhile, it takes the light $3.3 \mathrm{ps}$ to travel $1 \mathrm{~mm}$.

\section{Dark Matter and Particles Standard Model}

As discussed before it has seen, the mass of the electron is much heavier than this candidate particle by 2.5796 billion times, meanwhile, the proton weighs 4.7365 trillion $\left(4.7365 \times 10^{12}\right)$ dark matter particles. The atomic mass unit atmu $=0.93146 \mathrm{GeV} / \mathrm{c}^{2}$. Now let us compare the mass of the dark matter particle to the standard value of (atmu), leads to the conclusion that 4.7220 Trillion $\left(4.7220 \times 10^{12}\right)$ dark matter particles are required to construct 1 unity of atomic mass unit. In other words each dark matter particle weighs $\left(2.1177 \times 10^{-13}\right)$ atmu.

A proton has a mass of approximately $938 \mathrm{MeV} / \mathrm{c}^{2}$, of which the rest mass of its three valence quarks only contributes about $9.6 \mathrm{MeV} / \mathrm{c}^{2}$; much of the remainder can be attributed to the gluons quantum chromodynamics binding energy (QCBE). 
Based on the standard model [35], each proton is composed of 2 up quarks and 1 down quark as shown in Figure 1. Meanwhile, Figure 2 shows Neutron is composed of 1 up quark and 2 down quarks. It is desired now to compare the mass of the dark matter particle with the up quark $(2.4 \mathrm{MeV})$, down quark $(4.8 \mathrm{MeV})$, and the gluon $(928.4 \mathrm{MeV})$, respectively. The comparison leads to the following results: the up quark is equivalent to 12.167 billion dark matter particles $\left(12.167 \times 10^{9}\right)$, and the down quark mass matches 2.4334 billion $\left(24.334 \times 10^{9}\right)$ dark matter particles; meanwhile, the gluon is equivalent to 4.7065 Trillion of dark matter particles $\left(4.7065 \times 10^{12}\right)$.

$$
\begin{aligned}
1 \text { Proton }= & 2 \mathrm{u}+2 \mathrm{~d}+\text { Gloun Energy } \\
& =2 \times 2.4 \mathrm{MeV}+4.8 \mathrm{MeV}+\mathrm{QCBE} \\
& =9.6 \mathrm{MeV}+\mathrm{QCBE} \\
1 \text { Neutron } & =1 \mathrm{u}+2 \mathrm{~d}+\text { Gloun Energy } \\
& =2.4 \mathrm{MeV}+2 \times 4.8 \mathrm{MeV}+\mathrm{QCBE} \\
& =12 \mathrm{MeV}+\mathrm{QCBE}
\end{aligned}
$$

\section{Dark Energy and the Accelerated Expansion of the Universe}

The equivalent mass of the universe can be estimated based on the estimated age of the universe and the speed of light. One of the most of the acceptable estimated value of the age of the universe is considered as 13.7 billion years. Assume that after the Big Bang the universe is expanding such that it has a radius equivalent to the distance has been traveled by the light. Accordingly, it is possible to estimate the mass of the universe as follows

$$
\begin{aligned}
m & =\rho V=\rho(4 / 3) \pi r^{3}=\rho(4 / 3) \pi(c \cdot t)^{3} \\
& =9.9 \times 10^{-27}(4 / 3) \pi\left(2.998 \times 10^{8} \times 13.7 \times 10^{9} \times 365.25 \times 24 \times 3600\right)^{3}=9.0116 \times 10^{52} \mathrm{~kg}
\end{aligned}
$$

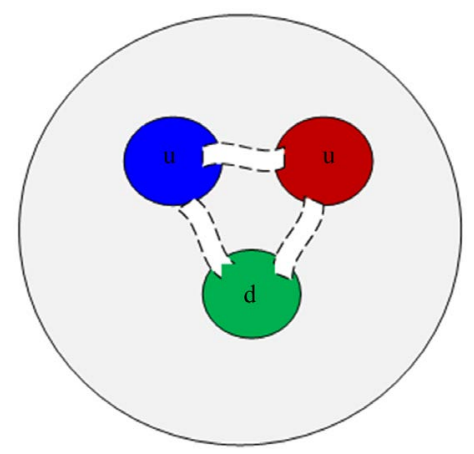

Figure 1. Schematics of the structure of the proton according to standard model.

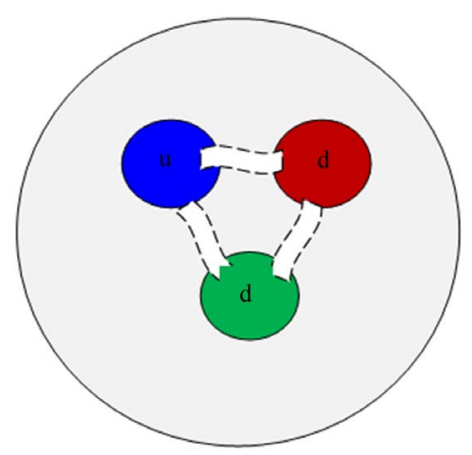

Figure 2. Schematics of the structure of the neutron to standard model. 
The equivalent mass of the universe can be presented in an equivalent number of protons and dark matter particles as follows: $5.3878 \times 10^{79}$ protons or $2.5519 \times 10^{92}$ dark matter particles. Based on the astronomical observation that the universe is expanding at an accelerating rate, based on the CMB observation that the average temperature of the sky is $2.73 \mathrm{~K}$ and by the virtue of the ideal equation of state then it can be assumed that the expansion process is isothermal (constant temperature). It is possible now to express the equation of state as

$$
P=N K_{B} \frac{T}{V}
$$

In thermodynamics [2], the work involved when an ideal changes from state 1 to state 2 is simply:

$$
W_{1 \rightarrow 2}=\int_{V_{1}}^{V_{2}} P \mathrm{~d} V=\int_{V_{1}}^{V_{2}} N K_{B} \frac{T}{V} \mathrm{~d} V=N K_{B} T \int_{V_{1}}^{V_{2}} \frac{1}{V} \mathrm{~d} V=N K_{B} T \ln \frac{V_{2}}{V_{1}}
$$

For an isothermal, reversible process, this integral equals the area under the relevant pressure-volume isotherm for an ideal gas. By convention, work is defined as the work the system does on its environment. As per Joule's Law, Internal energy is the function of absolute temperature. In isothermal process the temperature is constant. Hence, the internal energy is constant. Moreover, the net change in internal energy is zero. Based on the ideal gas law governed by (2), $P V=m R T=E=$ const., it yields that

$$
P=N k_{B} T \times \frac{1}{V}
$$

Since the universe is expanding with an homogenous CMB temperature of $2.73 \mathrm{~K}$, then it is desired no to express the pressure to volume rate of change as follows:

$$
\begin{aligned}
& \mathrm{d} P / \mathrm{d} V=\mathrm{d} / \mathrm{d} V\left(N k_{B} T \times \frac{1}{V}\right) \\
& \mathrm{d} P / \mathrm{d} V=-N k_{B} T \times \frac{1}{V^{2}} \\
& \mathrm{~d} P / \mathrm{d} V=\text { const } \times-\frac{1}{V^{2}}
\end{aligned}
$$

By the virtue of (29), it is concluded that the universe rate of change of pressure with respect of its volume is inversely proportional to the negative of squared volume $-1 / V^{2}$ as shown in Figure 3. Furthermore, since the dark energy pressure is so tiny small $8.8981 \times 10^{-10} \mathrm{~J} / \mathrm{m}^{3}$ but not zero, then it yields that the universe volume is not infinite and flat. Remember that the estimated current universe volume is

$(4 / 3) \pi\left(2.998 \times 10^{8} \times 13.7 \times 10^{9} \times 365.25 \times 24 \times 3600\right)^{3}$ which is equivalent to $9.1213 \times 10^{78} \mathrm{~m}^{3}$. Hence, the current universe pressure to volume rate of change $-1.155 \times 10^{-88} \mathrm{~N} / \mathrm{m}^{2} / \mathrm{m}^{3}$.

QGP Critical Temprature

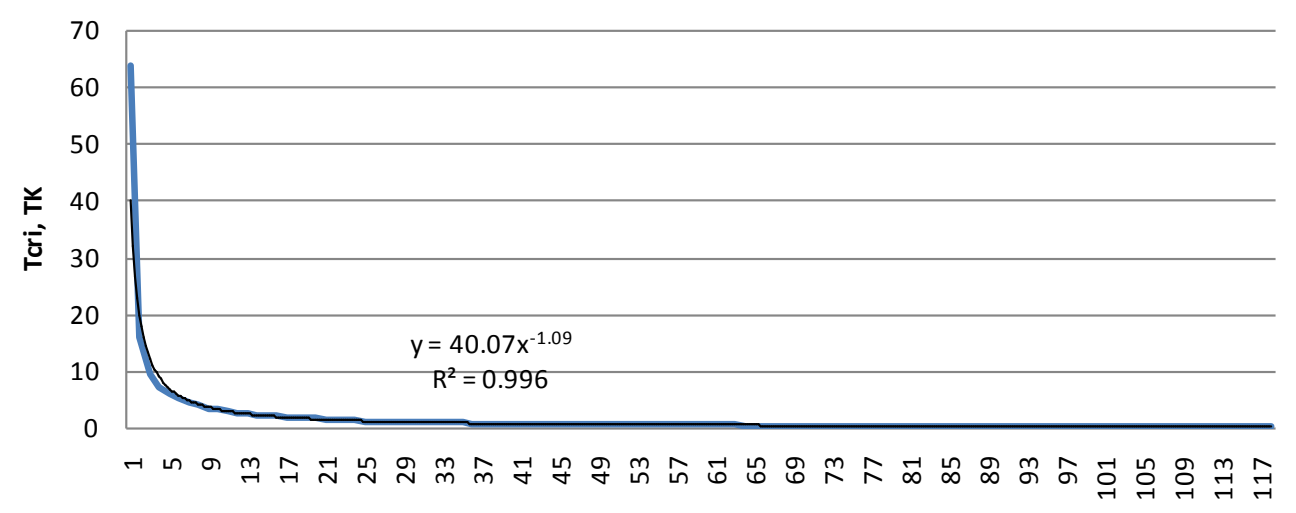

Figure 3. QGP critical temperature for natural elements of the periodic table. 


\section{The Generation of the Periodic Table Elements and Critical Temperature}

The critical temperature at which one entity would be generated is found to be equal to 64.478 Tetra Kelvin as addressed earlier in Section 3. Interestingly, the calculated critical temperature of the quark-gluon plasma is found to be proportional to the temperature generated by colliding heavy ions at the Relativistic Heavy Ion Collider (RHIC) and European Organization for Nuclear Research (CERN).

Recent analyses from the Relativistic Heavy Ion Collider (RHIC), a 2.4-mile-circumference (atom smasher) at the U.S. Department of Energy's (DOE) Brookhaven National Laboratory, establish that collisions of gold ions traveling at nearly the speed of light have created matter at a temperature of about 4 Trillion degrees Celsius- the hottest temperature ever reached in a laboratory, about 250,000 times hotter than the center of the Sun [27]-[31]. This temperature, based upon measurements by the PHENIX collaboration at RHIC, is higher than the temperature needed to melt protons and neutrons into plasma of quarks and gluons. These new temperature measurements, combined with other observations analyzed over nine years of operations by RHIC's four experimental collaborations - BRAHMS, PHENIX, PHOBOS, and STAR — indicate that RHIC's gold-gold collisions produce a freely flowing liquid composed of quarks and gluons. Such a substance, often referred to as quark-gluon plasma, or QGP, filled the universe a few microseconds after it came into existence 13.7 billion years ago. At RHIC, this liquid appears, and the quoted temperature is reached, in less time than it takes light to travel across a single proton.

The objective in this section is to determine the individual critical temperature of the quark-gluon plasma matter at which the elements of the Periodic Table are generated. The quark-gluon plasma (QGP) critical temperature of the elements of the Periodic Table Groups and Periods is calculated by the virtue of Equation (13):

$$
T=\frac{\rho_{\mathrm{E}}}{\rho_{\mathrm{N}} K_{B}}=6.4478 \times 10^{13} \frac{1}{\rho_{\mathrm{N}}} \mathrm{K}=\frac{64.478}{\rho_{\mathrm{N}}} \mathrm{TK} \text {. Based on the results demonstrated in Novel Periodic Table }
$$

displayed in Table 1 and Table 4, it can be seen that the highest critical temperature needed to generate quarkgluon plasma (QGP) from Hydrogen atom is 64 TK (5516 MeV), followed by $16.1 \mathrm{TK}$ (1389 MeV) needed to generate QGP from Helium, ended by the 0.219 TK $(18.9 \mathrm{MeV})$ to produce QGP from the last Periodic undiscovered yet element. It is interesting to know that the critical temperatures at which QGP is generated out of Gold atom and Uranium atom are $0.327 \mathrm{TK}(28.8 \mathrm{MeV})$ and $0.271(23.4 \mathrm{MeV})$, respectively. The average QGP critical temperature of all natural elements is figured to be $1.627 \mathrm{TK}(18.9 \mathrm{MeV})$ as demonstrated in Table 1.

Based on these obtained primary results of QGP critical temperatures, it is interesting to test the QGP critical temperature trend of the elements of the Periodic Table Groups and Periods. It is figured out that the overall trend is of negative power regression $y=40.07 x^{-1.099}$ (TK) in Temperature scale and $y=3455.1 x^{-1.099}(\mathrm{MeV})$ in energy scale as shown in Figure 3 and Figure 4, respectively. This hints how was the trend at which the elements were created after the Big Bang. The QGP critical temperature is decreasing dramatically going down from Hydrogen, through Helium and ending by the Transition metals at Period 7.

Let us examine the QGP critical temperature of the Periods and the groups separately. The critical temperature of Periods is found to have either linear trend with negative slope or quadratic decreasing trend. The first Period takes the linear form, followed by a quadratic form for the second Period, followed by a linear trend for the third Period, followed by quadratic form for the fourth Period, and then followed by a linear trend for all successive Periods fifth sixth and the seventh. Please refer to Table 2 and Figure 5. It noticed that the negative slope is increasing by going down from the top Period to the bottom Period from slope of -47.867 to -0.00038 . The quadratic coefficient is also decreasing from 0.1078 in Period 2 to 0.0019 in Period 4.

On the other hand, let us now examine the QGP critical temperature of the groups. The critical temperature of groups is found to have negative power aggression trend. It is found out that both the power coefficient and negative exponent are decreasing as we go from left side of the groups A to the right side groups A. Similarly, groups B are showing a QGP decreasing QGP critical temperature pattern. Please refer to Table 3, Figure 6 and Figure 7. It noticed that the negative slope is increasing by going down from the top Period to the bottom Period. For example Group 1A shows a power trend of $y=63.055 x^{-2.737}$ (TK) (or $y=10.356 x^{-1.902}$ (TK) * excluding $\mathrm{H})$, and ends with $y=16.077 x^{-2.199}$ (TK) (or $y=3.7873 x^{-1.532}$ (TK) Excluding He) as displayed in Figure 6. Likely, Groups B (starting with 4B, 5B, 6B, 7B, 8B1, 8B2, 8B3, 1B and 2B) demonstrate negative power regression starting with $y=1.4459 x^{-1.247}$ and ending with $y=1.0516 x^{-1.067}$ (TK). The exception to this pattern is Group 3B which shows linearly decreasing trend beginning with $y=1.5012 x^{-1.136}$ (TK) and terminating with behavior $y=-0.1224 x+0.4909$. The QGP Critical Temperatures of Lanthanide and Actinide Series are shown 
Table 1. Novel projected periodic table of quark-gluon plasma critical temperature of natural elements.

\begin{tabular}{|c|c|c|c|c|c|c|c|c|c|c|c|c|c|c|c|c|c|c|}
\hline Group & 1 & 2 & 3 & 4 & 5 & 6 & 7 & 8 & 9 & 10 & 11 & 12 & 13 & 14 & 15 & 16 & 17 & 18 \\
\hline \multicolumn{19}{|l|}{ Period } \\
\hline 1 & $\begin{array}{c}1 \\
\mathbf{H} \\
64.0\end{array}$ & & & & & & & & & & & & & & & & & $\begin{array}{c}2 \\
\mathbf{H e} \\
16.1\end{array}$ \\
\hline 2 & $\begin{array}{c}3 \\
\mathbf{L i} \\
9.29\end{array}$ & $\begin{array}{c}4 \\
\text { Be } \\
7.15\end{array}$ & & & & & & & & & & & $\begin{array}{c}5 \\
\text { B } \\
5.97\end{array}$ & $\begin{array}{c}6 \\
\mathbf{C} \\
5.37\end{array}$ & $\begin{array}{c}7 \\
\mathbf{N} \\
4.60\end{array}$ & $\begin{array}{c}8 \\
\mathbf{O} \\
4.03\end{array}$ & $\begin{array}{c}9 \\
\mathbf{F} \\
3.39\end{array}$ & $\begin{array}{c}10 \\
\mathbf{N e} \\
3.20\end{array}$ \\
\hline 3 & $\begin{array}{c}11 \\
\mathbf{N a} \\
2.80\end{array}$ & $\begin{array}{c}12 \\
\mathbf{M g} \\
2.65\end{array}$ & & & & & & & & & & & $\begin{array}{c}13 \\
\mathbf{A l} \\
2.39\end{array}$ & $\begin{array}{c}14 \\
\mathbf{S i} \\
2.30\end{array}$ & $\begin{array}{c}15 \\
\mathbf{P} \\
2.08\end{array}$ & $\begin{array}{c}16 \\
\mathbf{S} \\
2.01\end{array}$ & $\begin{array}{c}17 \\
\mathbf{C l} \\
1.82\end{array}$ & $\begin{array}{c}18 \\
\mathbf{A r} \\
1.61\end{array}$ \\
\hline 4 & $\begin{array}{c}19 \\
\mathbf{K} \\
1.65\end{array}$ & $\begin{array}{c}20 \\
\mathbf{C a} \\
1.60\end{array}$ & $\begin{array}{c}21 \\
\text { Sc } \\
1.434\end{array}$ & $\begin{array}{c}22 \\
\mathbf{T i} \\
1.35\end{array}$ & $\begin{array}{c}23 \\
\mathbf{V} \\
1.27\end{array}$ & $\begin{array}{c}24 \\
\mathbf{C r} \\
1.24\end{array}$ & $\begin{array}{c}25 \\
\text { Mn } \\
1.17\end{array}$ & $\begin{array}{c}26 \\
\mathbf{F e} \\
1.15\end{array}$ & $\begin{array}{c}27 \\
\text { Co } \\
1.09\end{array}$ & $\begin{array}{c}28 \\
\mathbf{N i} \\
1.10\end{array}$ & $\begin{array}{c}29 \\
\mathbf{C u} \\
1.01\end{array}$ & $\begin{array}{c}30 \\
\mathbf{Z n} \\
0.986\end{array}$ & $\begin{array}{c}31 \\
\mathbf{G a} \\
0.924\end{array}$ & $\begin{array}{c}32 \\
\mathbf{G e} \\
0.887\end{array}$ & $\begin{array}{c}33 \\
\text { As } \\
0.860\end{array}$ & $\begin{array}{c}34 \\
\mathbf{S e} \\
0.816\end{array}$ & $\begin{array}{cc} & 35 \\
& \mathbf{B r} \\
6 \quad 0.807\end{array}$ & $\begin{array}{c}36 \\
\mathbf{K r} \\
0.769\end{array}$ \\
\hline 5 & $\begin{array}{c}37 \\
\mathbf{R b} \\
0.754\end{array}$ & $\begin{array}{c}38 \\
\mathbf{S r} \\
0.736\end{array}$ & $\begin{array}{c}39 \\
\mathbf{Y} \\
6 \\
0.725\end{array}$ & $\begin{array}{cc} & 40 \\
& \mathbf{Z r} \\
5 & 0.707\end{array}$ & $\begin{array}{c}41 \\
\mathbf{N b} \\
7 \quad 0.694\end{array}$ & $\begin{array}{c}42 \\
\text { Mo } \\
0.672\end{array}$ & $\begin{array}{c}43 \\
\text { Tc } \\
0.658\end{array}$ & $\begin{array}{c}44 \\
\text { Ru } \\
0.638\end{array}$ & $\begin{array}{c}45 \\
\text { Rh } \\
0.627\end{array}$ & $\begin{array}{c}46 \\
\text { Pd } \\
0.606\end{array}$ & 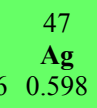 & $\begin{array}{c}48 \\
\text { Cd } \\
0.574\end{array}$ & $\begin{array}{c}49 \\
\text { In } \\
0.562\end{array}$ & $\begin{array}{c}50 \\
\text { Sn } \\
0.543\end{array}$ & $\begin{array}{c}51 \\
\text { Sb } \\
0.530\end{array}$ & $\begin{array}{c}52 \\
\text { Te } \\
0.505\end{array}$ & $\begin{array}{cc} & 53 \\
& \mathbf{I} \\
5 & 0.508\end{array}$ & $\begin{array}{c}54 \\
\mathbf{X e} \\
0.491\end{array}$ \\
\hline 6 & $\begin{array}{c}55 \\
\text { Cs } \\
0.485\end{array}$ & $\begin{array}{c}56 \\
\mathbf{B a} \\
0.470\end{array}$ & $*$ & $\begin{array}{c}72 \\
\text { Hf } \\
0.361\end{array}$ & $\begin{array}{c}73 \\
\text { Ta } \\
1 \quad 0.356\end{array}$ & $\begin{array}{c}74 \\
\mathbf{W} \\
0.350\end{array}$ & $\begin{array}{c}75 \\
\operatorname{Re} \\
0.346\end{array}$ & $\begin{array}{c}76 \\
\text { Os } \\
0.339\end{array}$ & $\begin{array}{c}77 \\
\mathbf{I r} \\
0.335\end{array}$ & $\begin{array}{c}78 \\
\mathbf{P t} \\
0.331\end{array}$ & $\begin{array}{c}79 \\
\text { Au } \\
1 \quad 0.327\end{array}$ & $\begin{array}{c}80 \\
\mathbf{H g} \\
0.321\end{array}$ & $\begin{array}{c}81 \\
\text { TI } \\
0.315\end{array}$ & $\begin{array}{c}82 \\
\mathbf{P b} \\
0.311\end{array}$ & $\begin{array}{c}83 \\
\mathbf{B i} \\
0.309\end{array}$ & $\begin{array}{c}84 \\
\text { Po } \\
0.309\end{array}$ & $\begin{array}{cc} & 85 \\
& \text { At } \\
9 & 0.307\end{array}$ & $\begin{array}{c}86 \\
\text { Rn } \\
0.290\end{array}$ \\
\hline 7 & $\begin{array}{c}87 \\
\mathbf{F r} \\
0.289\end{array}$ & $\begin{array}{c}88 \\
\mathbf{R a} \\
0.285\end{array}$ & $* *$ & $\begin{array}{c}104 \\
\mathbf{R f} \\
0.241\end{array}$ & $\begin{array}{c}105 \\
\mathbf{D b} \\
1 \quad 0.241\end{array}$ & $\begin{array}{c}106 \\
\mathbf{S g} \\
0.238\end{array}$ & $\begin{array}{c}107 \\
\text { Bh } \\
0.237\end{array}$ & $\begin{array}{c}108 \\
\mathbf{H s} \\
0.239\end{array}$ & $\begin{array}{c}109 \\
\text { Mt } \\
0.234\end{array}$ & $\begin{array}{c}110 \\
\text { Ds } \\
+\quad 0.229\end{array}$ & $\begin{array}{c}111 \\
\mathbf{R g} \\
0.226\end{array}$ & $\begin{array}{c}112 \\
\mathbf{C n} \\
0.226\end{array}$ & $\begin{array}{c}113 \\
\text { Uut } \\
0.227\end{array}$ & $\begin{array}{c}114 \\
\text { Fl } \\
0.223\end{array}$ & $\begin{array}{c}115 \\
\text { Uup } \\
0.224\end{array}$ & $\begin{array}{c}116 \\
\mathbf{L v} \\
+\quad 0.220\end{array}$ & $\begin{array}{cc}117 \\
\text { Uus } \\
0.219\end{array}$ & $\begin{array}{c}118 \\
\text { Uuo } \\
0.219\end{array}$ \\
\hline \multicolumn{2}{|c|}{$\begin{array}{l}\text { Lanthanide } \\
\text { Series* }\end{array}$} & $\begin{array}{c}57 \\
\mathbf{L a} \\
0.464\end{array}$ & $\begin{array}{c}58 \\
\mathbf{C e} \\
0.460\end{array}$ & $\begin{array}{c}59 \\
\mathbf{P r} \\
0.457\end{array}$ & $\begin{array}{c}60 \\
\text { Nd } \\
0.447\end{array}$ & $\begin{array}{c}61 \\
\text { Pm } \\
0.445\end{array}$ & $\begin{array}{c}62 \\
\text { Sm } \\
0.429\end{array}$ & $\begin{array}{c}63 \\
\text { Eu } \\
0.424\end{array}$ & & $\begin{array}{l}64 \\
\text { Gd } \\
410\end{array}$ & $\begin{array}{c}65 \\
\text { Tb } \\
0.406\end{array}$ & $\begin{array}{c}66 \\
\text { Dy } \\
0.397\end{array}$ & $\begin{array}{c}67 \\
\text { Ho } \\
0.391\end{array}$ & $\begin{array}{c}68 \\
\mathbf{E r} \\
0.385\end{array}$ & & $\begin{array}{l}69 \\
\Gamma \mathbf{m} \\
382\end{array}$ & $\begin{array}{c}70 \\
\mathbf{Y b} \\
0.373\end{array}$ & $\begin{array}{c}71 \\
\text { Lu } \\
0.369\end{array}$ \\
\hline \multicolumn{2}{|c|}{$\begin{array}{l}\text { Actinide } \\
\text { Series** }\end{array}$} & $\begin{array}{c}89 \\
\text { Ac } \\
0.284\end{array}$ & $\begin{array}{c}90 \\
\text { Th } \\
0.278\end{array}$ & $\begin{array}{c}91 \\
\mathbf{P a} \\
0.279\end{array}$ & $\begin{array}{c}92 \\
\mathbf{U} \\
0.271\end{array}$ & $\begin{array}{c}93 \\
\mathbf{N p} \\
0.272\end{array}$ & $\begin{array}{c}94 \\
\mathbf{P u} \\
0.264\end{array}$ & $\begin{array}{c}95 \\
\text { Am } \\
0.265\end{array}$ & & $\begin{array}{l}96 \\
\mathrm{Cm} \\
261\end{array}$ & $\begin{array}{c}97 \\
\text { Bk } \\
0.261\end{array}$ & $\begin{array}{c}98 \\
\text { Cf } \\
0.257\end{array}$ & $\begin{array}{c}99 \\
\text { Es } \\
0.255\end{array}$ & $\begin{array}{c}100 \\
\text { Fm } \\
0.250\end{array}$ & & $\begin{array}{l}01 \\
\text { Md } \\
250\end{array}$ & $\begin{array}{c}102 \\
\text { No } \\
0.249\end{array}$ & $\begin{array}{c}103 \\
\mathbf{L r} \\
0.246\end{array}$ \\
\hline
\end{tabular}

\begin{tabular}{ccc}
\hline & Alkali metals & Lanthanides \\
& Alkaline earth metals & Actinides \\
& Transition metals & Nonmetals \\
& Post-transition metals & Halogens \\
& Metalloid & Noble gases \\
\hline $\mathbf{1}$ & & Atomic Number \\
\hline $\mathbf{6 4 . 0}$ & \multicolumn{2}{c}{ Quark-Gluon Critical Temperature (TK) } \\
\hline
\end{tabular}

QGP Critical Energy

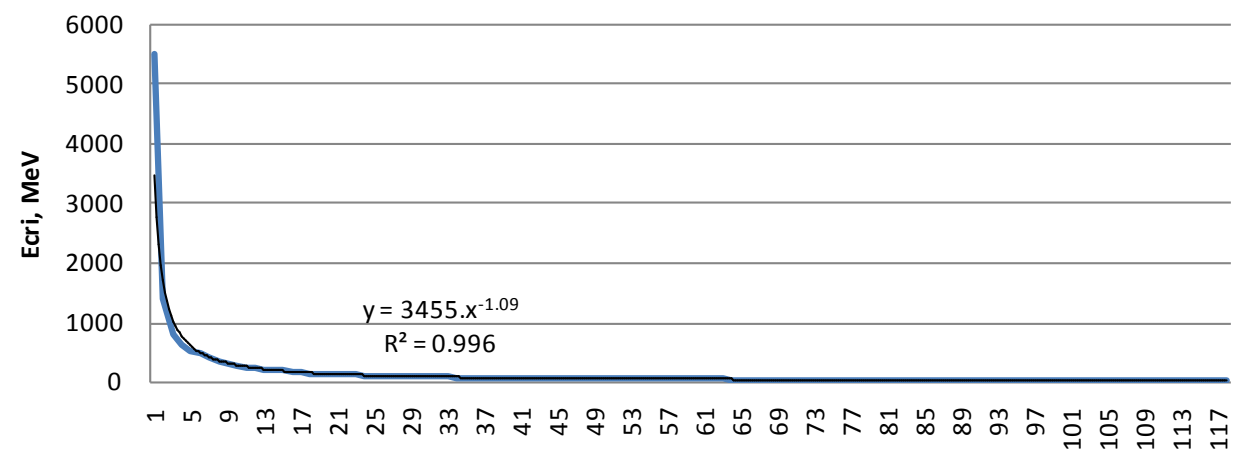

Figure 4. QGP critical energy for natural elements of the periodic table. 
Table 2. The regression trend of QGP critical temperature for periods of the periodic table.

\begin{tabular}{ccc}
\hline Period & Equation & $\mathbf{R}^{\mathbf{2}}$ \\
\hline Period 1 & $y=-47.867 x+111.84$ & 1 \\
Period 2 & $y=0.1078 x^{2}-1.7808 x+10.64$ & 0.986 \\
Period 3 & $y=-0.1649 x+2.9508$ & 0.9904 \\
Period 4 & $y=0.0019 x^{2}-0.085 x+1.7029$ & 0.9849 \\
Period 5 & $y=-0.0159 x+0.7691$ & 0.997 \\
Period 6 & $y=-0.0156 x+0.5008 ; y=-0.0074 x+0.4749 ; y=-0.0046 x+0.364$ & $1 ; 0.9982 ; 0.98$ \\
Period 7 & $y=-0.0038 x+0.293 ; y=-0.0026 x+0.2839 ; y=-0.0017 x+0.2436 ;$ & $1 ; 0.9726 ; 0.9552$ \\
\hline
\end{tabular}

Table 3. The regression trend of QGP critical temperature for the groups of the periodic table.

\begin{tabular}{|c|c|c|}
\hline Group & Equation & $\mathbf{R}^{2}$ \\
\hline Group 1A & $\begin{array}{rl}y & =63.055 x^{-2.737} \\
y & =10.356 x^{-1.902^{*}} \\
* & * \text { (Excluding H) }\end{array}$ & $\begin{array}{l}\mathrm{R}^{2}=0.9981 \\
\mathrm{R}^{2}=0.987^{*}\end{array}$ \\
\hline Group 2A & $y=8.4232 x^{-1.776}$ & $\mathrm{R}^{2}=0.9771$ \\
\hline Group 3A & $y=6.9729 x^{-1.867}$ & $\mathrm{R}^{2}=0.9878$ \\
\hline Group 4A & $y=6.3518 x^{-1.821}$ & $\mathrm{R}^{2}=0.9854$ \\
\hline Group 5A & $y=5.4589 x^{-1.73}$ & $\mathrm{R}^{2}=0.9838$ \\
\hline Group 6A & $y=4.8691 x^{-1.669}$ & $\mathrm{R}^{2}=0.979$ \\
\hline Group 7A & $y=4.1472 x^{-1.566}$ & $\mathrm{R}^{2}=0.9737$ \\
\hline Group 8A & $\begin{array}{c}y=3.7873 x^{-1.532 \text { (Excluding He) }} \\
y=16.077 x^{-2.199}\end{array}$ & $\begin{array}{c}\mathrm{R}^{2}=0.9978 \\
\mathrm{R}^{2}=0.9794 * *\end{array}$ \\
\hline Group 3B & $\begin{aligned} y & =1.5012 x-1.136 \\
y=-0.1801 x+0.6443 \text { (Has been calculated with 3B) } & =-0.1823 x+0.6425 \\
y & =-0.1785 x+0.6361 \\
y & =-0.1761 x+0.6231 \\
y & =-0.1726 x+0.6173 \\
y & =-0.1646 x+0.5934 \\
y & =-0.159 x+0.5833 \\
y & =-0.149 x+0.559 \\
y & =-0.1447 x+0.5504 \\
y & =-0.1399 x+0.5367 \\
y & =-0.1351 x+0.526 \\
y & =-0.1346 x+0.5201 \\
y & =-0.1318 x+0.5134 \\
y & =-0.1236 x+0.4962 \\
y & =-0.1224 x+0.4909\end{aligned}$ & $\mathrm{R}^{2}=0.9864$ \\
\hline Group 4B & $y=1.4459 x^{-1.247}$ & $\mathrm{R}^{2}=0.9819$ \\
\hline Group 5B & $y=1.3652 x^{-1.206}$ & $\mathrm{R}^{2}=0.9779$ \\
\hline Group 6B & $y=1.3303 x^{-1.198}$ & $\mathrm{R}^{2}=0.9807$ \\
\hline Group 7B & $y=1.2625 x^{-1.161}$ & $\mathrm{R}^{2}=0.9779$ \\
\hline Group 8B1 & $y=1.2306 x^{-1.148}$ & $\mathrm{R}^{2}=0.9819$ \\
\hline Group 8B2 & $y=1.1743 x^{-1.122}$ & $\mathrm{R}^{2}=0.9776$ \\
\hline Group 8B3 & $y=1.1689 x^{-1.136}$ & $\mathrm{R}^{2}=0.9832$ \\
\hline Group 1B & $y=1.0883 x^{-1.077}$ & $\mathrm{R}^{2}=0.9763$ \\
\hline Group 2B & $y=1.0516 x^{-1.067}$ & $\mathrm{R}^{2}=0.9799$ \\
\hline
\end{tabular}


GQP Tcri. of Period 1

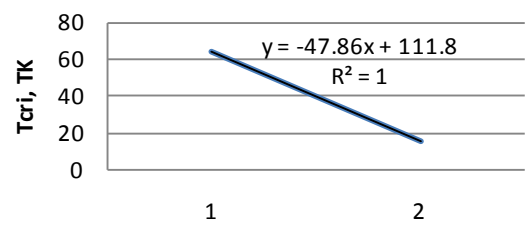

QGP Tcr. of Period 3

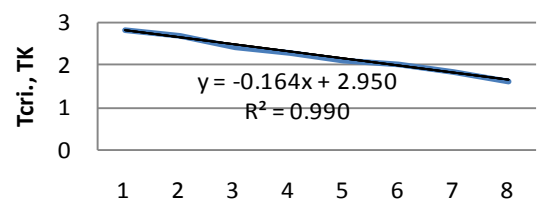

QGP Tcr. of Period 5

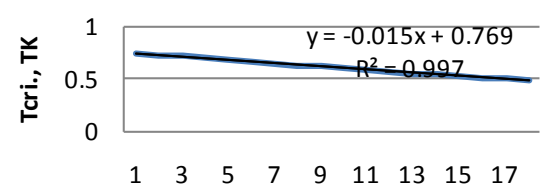

GQP Tcri. of Period 2

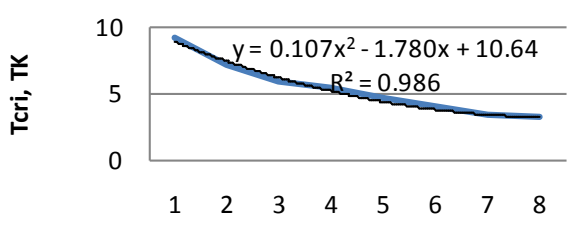

QGP Trci. of Period 4

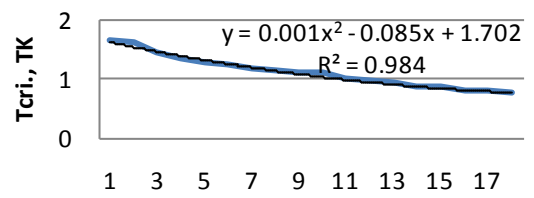

QGP Trci. of Period 6

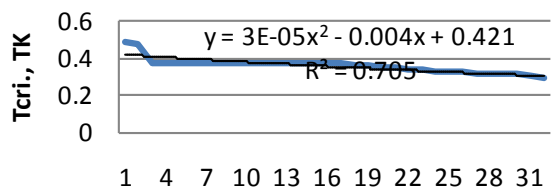

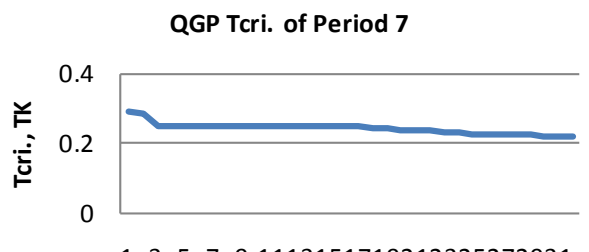

135791113151719212325272931

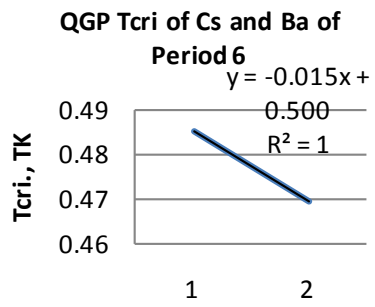

QGP Tcri of Fr and Ra of Period 7

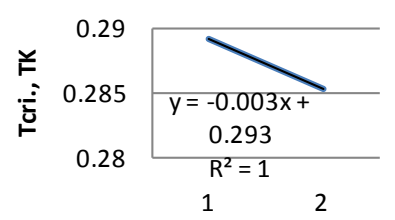

QGP Tcri. of Lanthanides Period 6

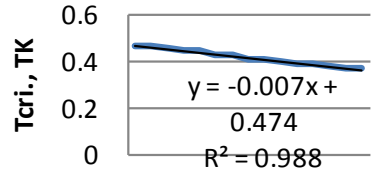

$\begin{array}{llllll}1 & 3 & 5 & 7 & 9 & 111315\end{array}$

QGP Tcri. of Actinide Period 7

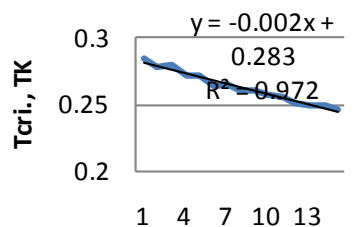

QGP Tcri. of Transmission Metals of Period 6

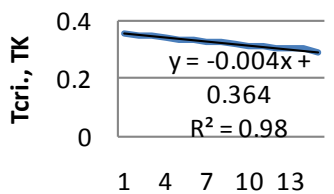

QGP Tcri. of Transmission Metals of Period 7

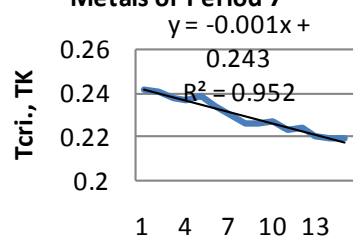

Figure 5. The regression trend of QGP critical temperature for periods of the periodic table.

in Figure 8. The pattern demonstrates a decreasing linear regression trend.

\section{Utilization of Quark-Gluon Plasma Energy and Its Phase Diagram: A Novel Millennium Power Plant}

It is estimated that the Hadron Epoch covers the time from $10^{-6}$ seconds to 1 second after the Big Bang [36]. The 
QGP Tcri. of Group 1A

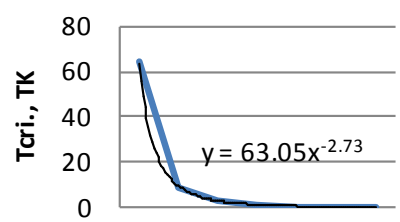

$\begin{array}{llllllll}1 & 2 & 3 & 4 & 5 & 6 & 7\end{array}$

\section{QGP Tcri. of Group 2A}

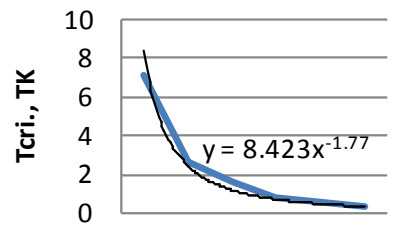

$\begin{array}{llllll}1 & 2 & 3 & 4 & 5 & 6\end{array}$

QGP Tcri. of Group 1A Excluding $\mathbf{H}$

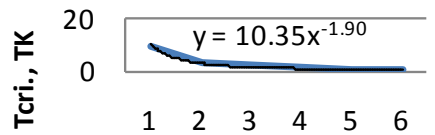

QGP Tcri. of Group 5A

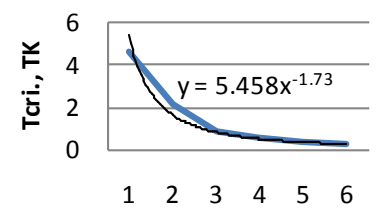

8QGP Tcri. of Group 8A

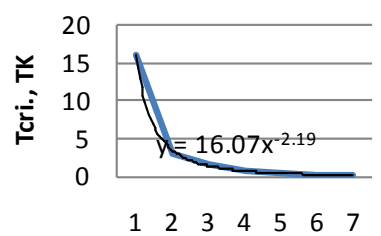

$\begin{array}{lllllllllllll}1 & 2 & 3 & 4 & 5 & 6 & 7\end{array}$
QGP Tcri. of Group 3A

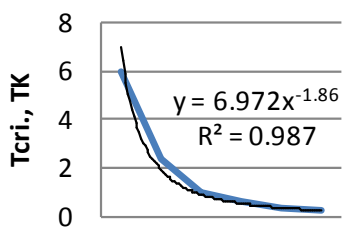

$\begin{array}{llllll}1 & 2 & 3 & 4 & 5 & 6\end{array}$
QGP Tcri. of Group 4A

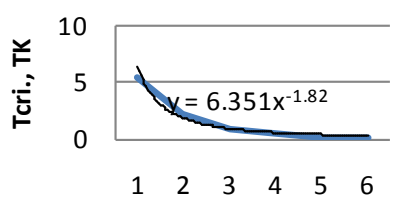

QGP Tcri. of Group 7A

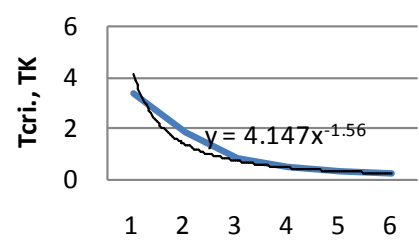

\section{QGP Tcri. of Group 5A}

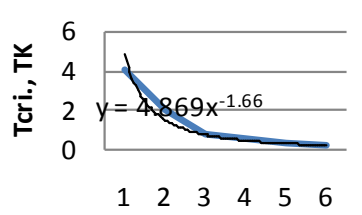

QGP Tcri. of Group 1A

Excluding $\mathrm{He}$

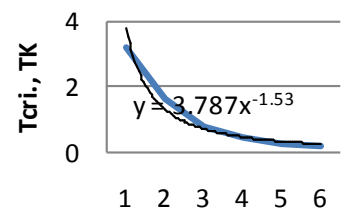

Figure 6. The regression trend of QGP critical temperature for groups a of the periodic table.

temperature during this epoch is estimated to decrease from $10^{13} \mathrm{~K}$ to $10^{10} \mathrm{~K}$. At $10^{-5}$ seconds, the temperature of the Universe was approximately $10^{12} \mathrm{~K}$. at which quarks combine to form protons and neutrons. Astrophysics predicts that Fermi melting point of quarks into quark-gluon plasma $\left(0.5-1.2 \times 10^{12} \mathrm{~K}\right)$ or higher. The results of this proposed analysis agree with such predictions. The phase diagram [28] of the quark-gluon plasma is a diagram at which the state of matter-energy. A new QGP phase diagram depicts the Hardon Epoch is proposed in Figure 9. The QGP phase diagram presents a critical point that separates the two phases: the hadron gas in which quarks are confined, and the quark-gluon plasma (QGP).

Experimental results on relative abundances of various hadron species created in gold-gold collisions at RHIC and their comparison with particle abundances realized in a simple hadron resonance gas (HRG) suggest that the transition back to ordinary hadrons, the so-called chemical freeze out, occurs at temperatures of about (160 - 170) $\mathrm{MeV}$ [32] [33]. Whether this freeze-out temperature is in any way related to the transition temperature in QCD is an important question that currently is studied extensively in large scale computer simulations. The simulations of the RBC-Bielefeld Collaboration performed on the QCDOC and apeNEXT computers hint at a larger transition temperature of about $190 \mathrm{MeV}$.

Based on this research analysis [37]-[42], it is found out that dark matter/dark energy behaves like an ideal fluid and there density are affected by the space temperature. Moreover, utilization of its energy can be achieved at high temperatures such as Fermi melting point. Hence $\rho_{N}$ is unity when the temperature $T$ is equal to $64 \times 10^{12} \mathrm{~K}$, then this temperature value is called the critical temperature. In other words at this temperature the $\rho_{N}=12.81 \times 10^{12}$ entities $/ \mathrm{m}^{3}$ of dark matter particles are unified (melted) to form quark-gluon plasma which work as the basic blocks of matter.

CMB and WMAP confirm that the density of dark matter/dark energy is uniform, homogeneous and constant 

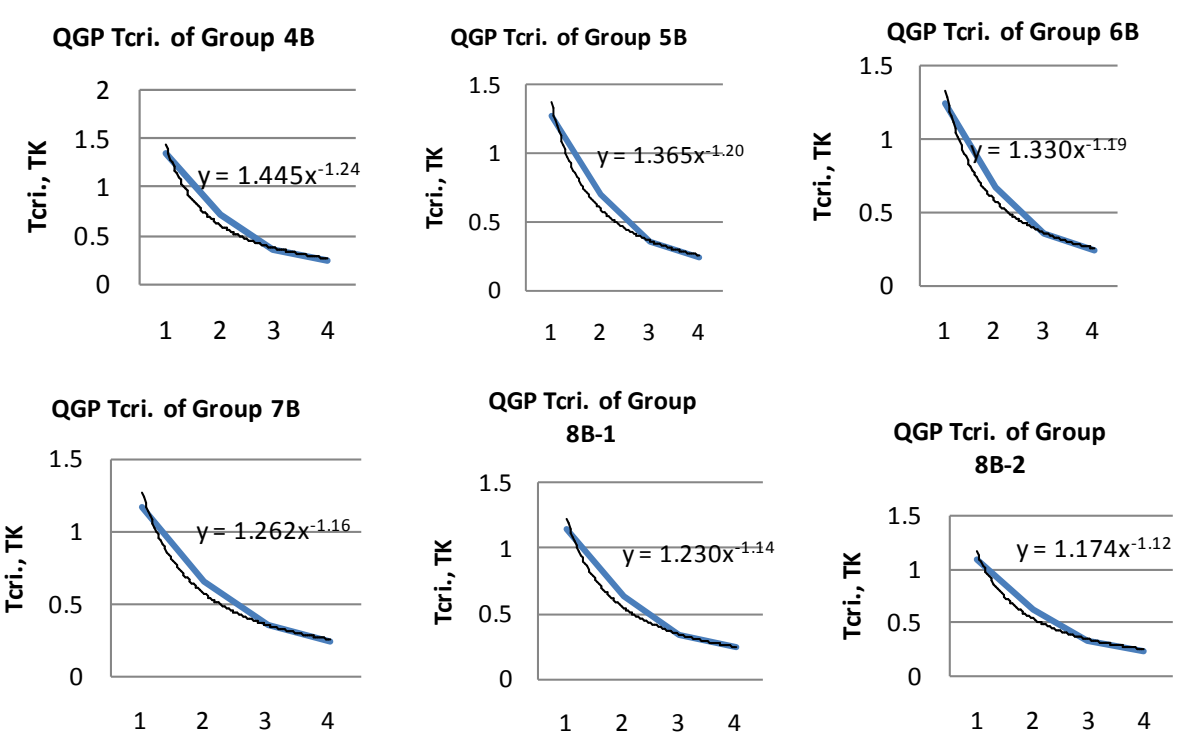

QGP Tcri. of Group 8B-3
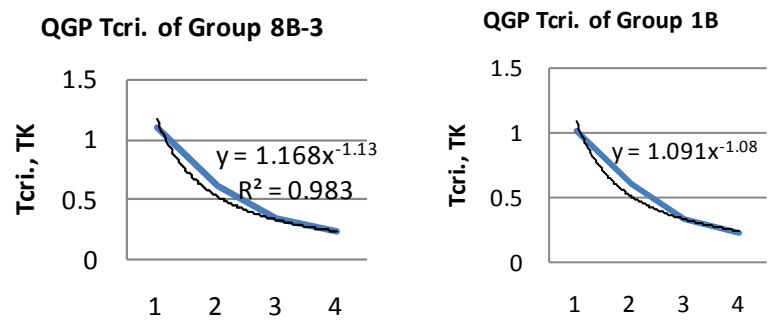

QGP Tcri. of Group 2B

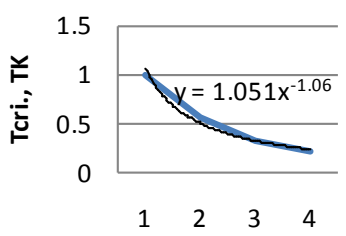

3QGP Tcri. of Group 3B

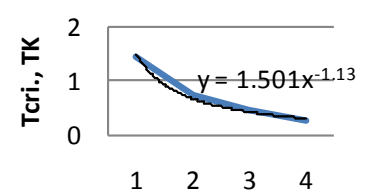

Figure 7. The regression trend of QGP critical temperature for groups B of the periodic table.

through the universe. Since the density is at the same order of the proton-nitron, then it is very possible that dark energy/dark matter is converted into quarks at this QGP critical temperature. Similar to Relativistic Heavy Ion Collider (RHIC), it is proposed here to generate quark-gluon plasma and utilize its associated energy. Based on Table 4, it can be seen that the generated energy QGP is ranging from $5516 \mathrm{MeV}$ associated with Hydrogen, $207 \mathrm{MeV}$ and $197 \mathrm{MeV}$ associated with Lead and Gold atoms, respectively.

Recent analyses from the Relativistic Heavy Ion Collider (RHIC), a 2.4-mile-circumference (atom smasher) at the U.S. Department of Energy's (DOE) Brookhaven National Laboratory, establish that collisions of gold ions traveling at nearly the speed of light have created matter at a temperature of about 4 trillion degrees Celsiusthe hottest temperature ever reached in a laboratory, about 250,000 times hotter than the center of the Sun [27]-[32]. This temperature, based upon measurements by the PHENIX collaboration at RHIC, is higher than the temperature needed to melt protons and neutrons into plasma of quarks and gluons. These new temperature measurements, combined with other observations analyzed over nine years of operations by RHIC's four experimental collaborations-BRAHMS, PHENIX, PHOBOS, and STAR - indicate that RHIC's gold-gold collisions produce a freely flowing liquid composed of quarks and gluons. Such a substance, often referred to as quark-gluon plasma, or QGP, filled the universe a few microseconds after it came into existence 13.7 billion years ago.

A novel Quark-Gluon Plasma power generation reactor is proposed in this work as displayed in Figure 10. It basically utilizes the output temperatures to heat up cold water and converts into a steam. The pressurized steam 
QGP Tcri. of Group La \& AC

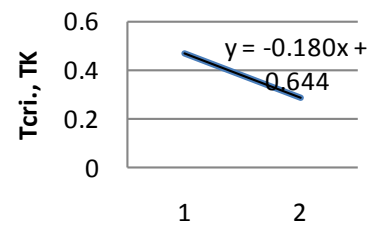

QGP Tcri. of Group Nd \& U

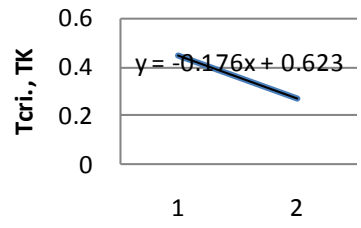

QGP Tcri. of Group Eu \& Am

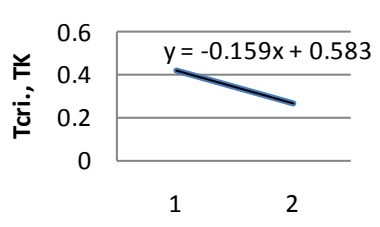

QGP Tcri. of Group Dy \& $\mathrm{Cr}$

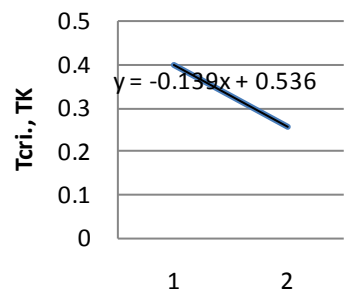

QGP Tcri. of Group Tm \& Md

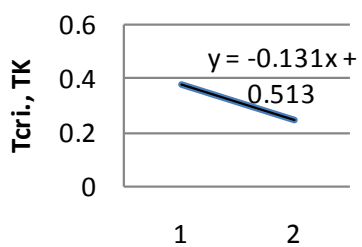

QGP Tcri. of Group $\mathrm{Ce} \& \mathrm{Th}$

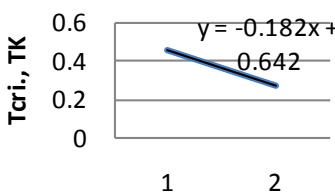

QGP Tcri. of Group Pm \& $\mathrm{Np}$

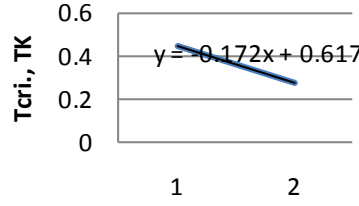

QGP Tcri. of Group Gd \&

$\mathrm{Cm}$

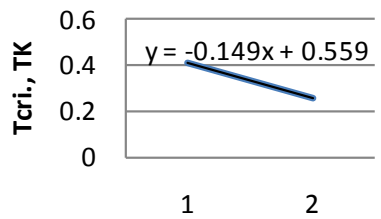

QGP Tcri. of Group Ho \& Es

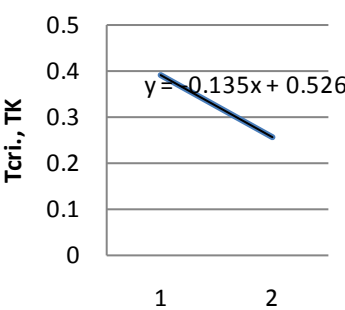

QGP Tcri. of Group Yb \&

No

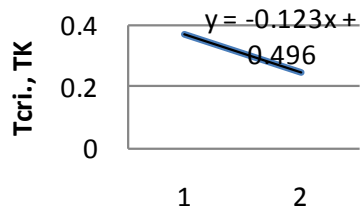

QGP Tcri. of Group Pr \& Pa

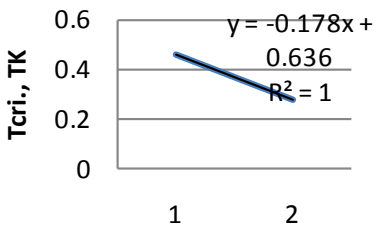

QGP Tcri. of Group Sm \&

$\mathrm{Pu}$

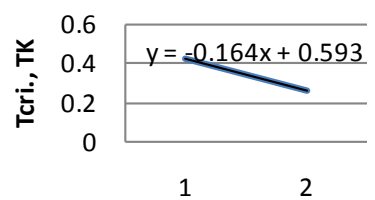

QGP Tcri. of Group Tb \& Bk

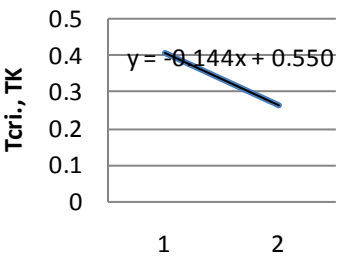

QGP Tcri. of Group Er \& Fm

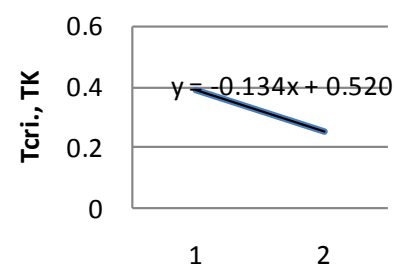

QGP Tcri. of Group Lu \& Lr

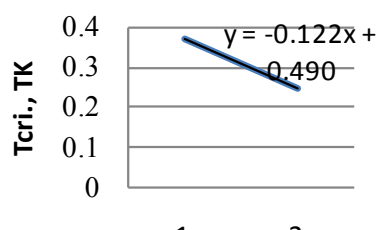

12

Figure 8. The regression trend of QGP critical temperature for the lanthanides and actinides of the periodic table.

will generate a high mechanical rotational at the turbines and then high induced electrical power at the electrical generators. It is remarkable to note here that the outcome energy of the QGP range [5516 to $18.9 \mathrm{MeV}$ ] would be much higher compared with the nuclear fission which generates $0.8 \mathrm{MeV}$ per atomic unit and $3.5 \mathrm{MeV}$ per atomic unit that can be produced from the nuclear fusion as tabulated in Table 5 .

\section{Conclusions}

In this work, a new physical interpretation of the state of matter of the quark-gluon as the most fundamental building blocks in nature. Such a model is derived based on the assumption that dark matter and dark energy 


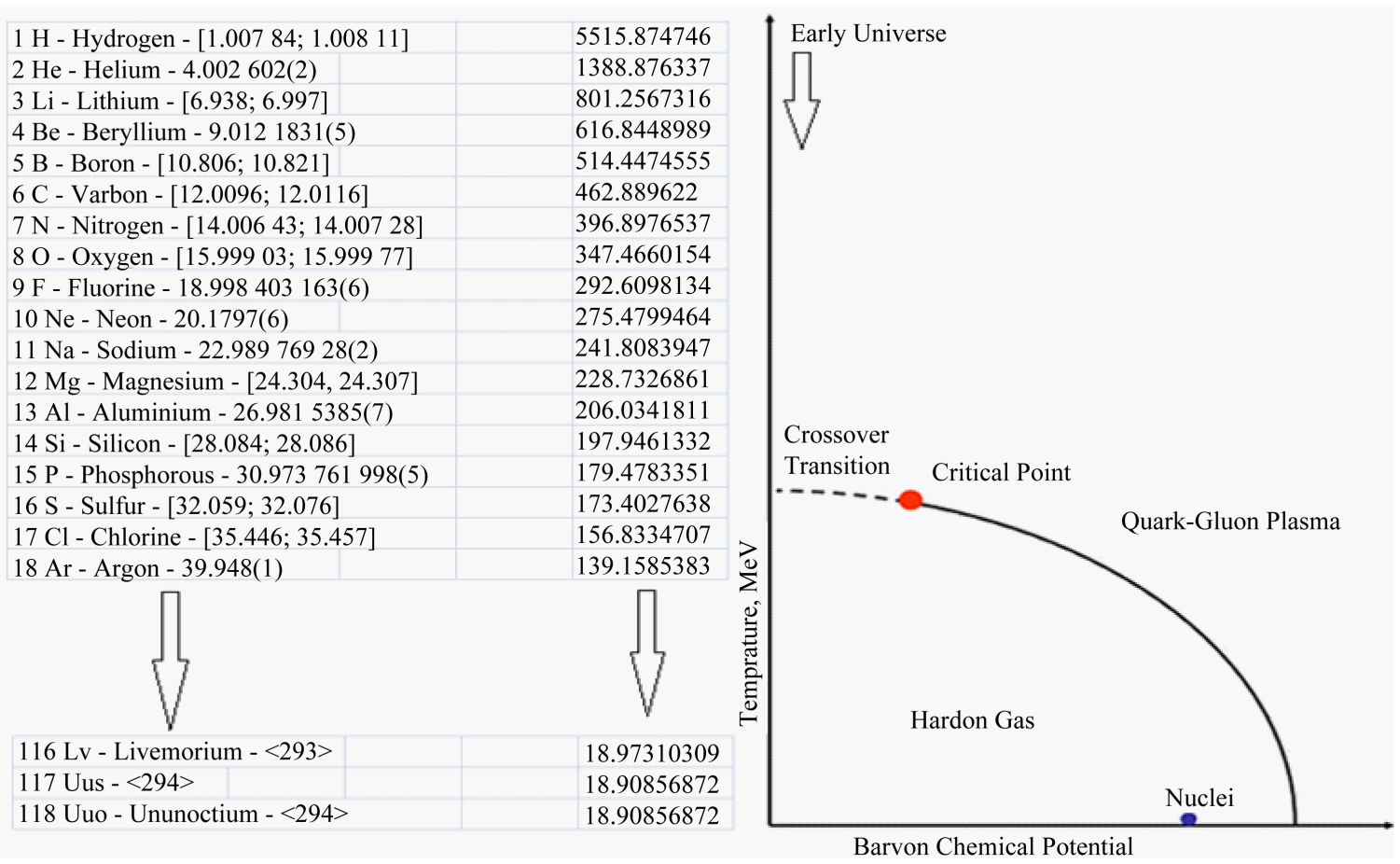

Figure 9. The QGP phase diagram and associated critical temperature.

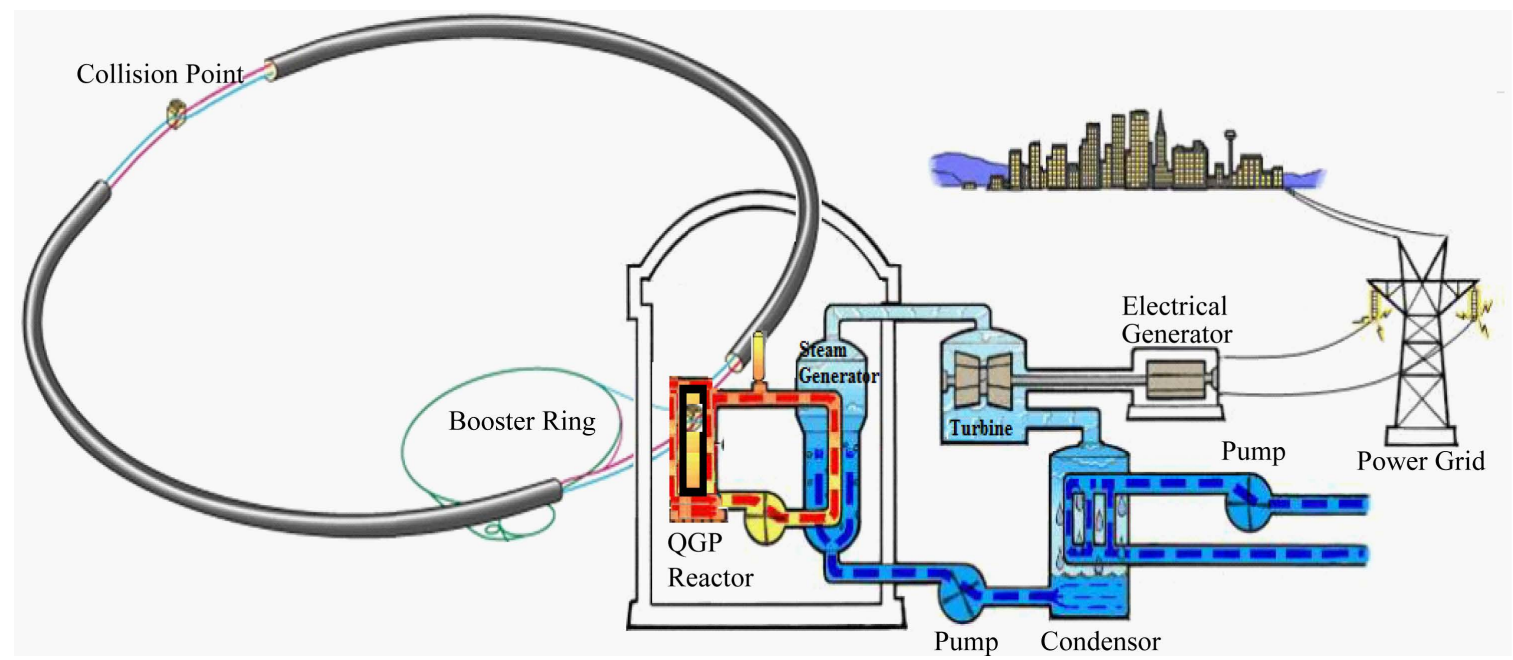

Figure 10. The novel QGP power generation plant.

behave as a perfect ideal fluid at extremely high temperature. By the virtue of Boltzmann constant of the ideal gas law and NASA's Cosmic Microwave Background Explorer (CMB) which estimate that the space has an average temperature close to $2.7251 \mathrm{Kelvin}$, then the equivalent mass-energy of the fundamental particle of the dark matter/dark energy is determined.

The average universe mass density of $9.91 \times 10^{-30} \mathrm{~g} / \mathrm{cm}^{3}$ is equivalent to only 5.9 protons per cubic meter or $8.8981 \times 10^{-10} \mathrm{~J} / \mathrm{m}^{3}=5.5302 \times 10^{9} \mathrm{eV} / \mathrm{m}^{3}=5.5302 \mathrm{GeV} / \mathrm{m}^{3}$. The temperature at which one entity will be generated is called in this work as the critical temperature and equals to $64 \times 10^{12}$ Kelvin. The calculated critical temperature at which 5.9 protons per cubic meter are produced is found to be $10.929 \times 10^{12} \mathrm{~K}$. The equivalent mass of dark matter particle, its mean life time and frequency is further determined.

Based on the standard model, the gluon energy that bonds quarks of protons and the quarks of the neutron is investigated. Each proton quark is tied by $928.4 \mathrm{MeV}$ gluon energy, Meanwhile, each proton quark is tied by 
Table 4. Projected values of quark-gluon plasma critical temperature of natural elements.

\begin{tabular}{|c|c|c|c|}
\hline Element & AW (Atomic Weight) & Equivalent Energy (MeV) & Critical Temperature (TK) \\
\hline $1 \mathrm{H}$ - Hydrogen - [1.007 84; 1.008 11] & 1.00784 & 5515.874746 & 63.97642483 \\
\hline $2 \mathrm{He}$ - Helium - 4.002 602(2) & 4.002602 & 1388.876337 & 16.10902108 \\
\hline 3 Li - Lithium - [6.938; 6.997] & 6.938 & 801.2567316 & 9.293456327 \\
\hline 4 Be - Beryllium - 9.012 1831(5) & 9.01218315 & 616.8448989 & 7.154537244 \\
\hline 5 B - Boron - $[10.806 ; 10.821]$ & 10.806 & 514.4474555 & 5.966870257 \\
\hline 6 C - Carbon - $[12.0096 ; 12.0116]$ & 12.0096 & 462.889622 & 5.368871569 \\
\hline $7 \mathrm{~N}$ - Nitrogen - [14.006 43; 14.00728$]$ & 14.00643 & 396.8976537 & 4.603457126 \\
\hline 8 O - Oxygen - [15.999 03; 15.99977$]$ & 15.99903 & 347.4660154 & 4.030119326 \\
\hline $9 \mathrm{~F}$ - Fluorine - 18.998403 163(6) & 18.99840316 & 292.6098134 & 3.393864181 \\
\hline $10 \mathrm{Ne}-\mathrm{Neon}-20.1797(6)$ & 20.17976 & 275.4799464 & 3.195181707 \\
\hline $11 \mathrm{Na}$ - Sodium - $22.98976928(2)$ & 22.98976928 & 241.8083947 & 2.804638846 \\
\hline $12 \mathrm{Mg}$ - Magnesium - [24.304, 24.307] & 24.304 & 228.7326861 & 2.652978934 \\
\hline $13 \mathrm{Al}$ - Aluminium - 26.981 5385(7) & 26.98153857 & 206.0341811 & 2.389708053 \\
\hline $14 \mathrm{Si}$ - Silicon - $[28.084 ; 28.086]$ & 28.084 & 197.9461332 & 2.29589802 \\
\hline 15 P - Phosphorus - 30.973761 998(5) & 30.973762 & 179.4783341 & 2.081697406 \\
\hline $16 \mathrm{~S}-$ Sulfur - $[32.059 ; 32.076]$ & 32.059 & 173.4027638 & 2.011229296 \\
\hline $17 \mathrm{Cl}$ - Chlorine - $[35.446 ; 35.457]$ & 35.446 & 156.8334707 & 1.819048694 \\
\hline $18 \mathrm{Ar}-$ Argon - 39.948(1) & 39.9481 & 139.1585383 & 1.614044222 \\
\hline $19 \mathrm{~K}$ - Potassium - 39.0983(1) & 39.09831 & 142.1831073 & 1.649124988 \\
\hline $20 \mathrm{Ca}$ - Calcium - 40.078(4) & 40.0784 & 138.7061161 & 1.608796758 \\
\hline $21 \mathrm{Sc}$ - Scandium - 44.955 908(5) & 44.9559085 & 123.657143 & 1.434249738 \\
\hline $22 \mathrm{Ti}$ - Titanium - 47.867(1) & 47.8671 & 116.1365365 & 1.347021232 \\
\hline $23 \mathrm{~V}$ - Vanadium - 50.9415(1) & 50.94151 & 109.1274916 & 1.265726124 \\
\hline $24 \mathrm{Cr}$ - Chromium - 51.9961(6) & 51.99616 & 106.9140337 & 1.240053112 \\
\hline $25 \mathrm{Mn}$ - Manganese - 54.938 044(3) & 54.9380443 & 101.1888806 & 1.17364935 \\
\hline $26 \mathrm{Fe}$ - Iron - $55.845(2)$ & 55.8452 & 99.54515704 & 1.154584458 \\
\hline 27 Co - Cobalt - 58.933 194(4) & 58.9331944 & 94.32916815 & 1.09408629 \\
\hline $28 \mathrm{Ni}$ - Nickel 58.6934(4) & 58.69344 & 94.71448946 & 1.098555477 \\
\hline $29 \mathrm{Cu}$ - Copper - 63.546(3) & 63.5463 & 87.48139867 & 1.014661751 \\
\hline $30 \mathrm{Zn}-\mathrm{Zinc}-65.38(2)$ & 65.382 & 85.02522413 & 0.986173565 \\
\hline $31 \mathrm{Ga}$ - Gallium - 69.723(1) & 69.7231 & 79.7313832 & 0.924772421 \\
\hline $32 \mathrm{Ge}$ - Germanium - 72.630(8) & 72.6308 & 76.53941859 & 0.887750101 \\
\hline 33 As - Arsenic - 74.921 595(6) & 74.9215956 & 74.19915659 & 0.860606338 \\
\hline $34 \mathrm{Se}$ - Selenium - 78.971(8) & 78.9718 & 70.39372541 & 0.816468663 \\
\hline $35 \mathrm{Br}$ - Bromine - [79.901, 79.907] & 79.901 & 69.57508922 & 0.80697363 \\
\hline $36 \mathrm{Kr}$ - Krypton - 83.798(2) & 83.7982 & 66.33936295 & 0.769443735 \\
\hline 37 Rb - Rubidium - 85.4678(3) & 85.46783 & 65.04341112 & 0.754412508 \\
\hline $38 \mathrm{Sr}$ - Strontium - 87.62(1) & 87.621 & 63.44505546 & 0.73587382 \\
\hline 39 Y - Yttrium - $88.90584(2)$ & 88.905842 & 62.52816552 & 0.725239181 \\
\hline $40 \mathrm{Zr}$ - Zirconium - 91.224(2) & 91.2242 & 60.93908419 & 0.706808062 \\
\hline
\end{tabular}




\section{Continued}

\begin{tabular}{|c|c|c|c|}
\hline $41 \mathrm{Nb}$ - Niobium - 92.906 37(2) & 92.906372 & 59.83571508 & 0.694010525 \\
\hline 42 Mo - Molybdenum - 95.95(1) & 95.951 & 57.93706375 & 0.671988828 \\
\hline $43 \mathrm{Tc}-$ Technetium $-<98>$ & 98 & 56.72570616 & 0.657938776 \\
\hline $44 \mathrm{Ru}$ - Ruthenium - 101.07(2) & 101.072 & 55.00157515 & 0.63794127 \\
\hline 45 Rh - Rhodium - 102.905 50(2) & 102.905502 & 54.02159356 & 0.626574855 \\
\hline 46 Pd - Palladium - 106.42(1) & 106.421 & 52.237051 & 0.60587666 \\
\hline $47 \mathrm{Ag}$ - Silver - 107.8682(2) & 107.86822 & 51.53620968 & 0.597747882 \\
\hline $48 \mathrm{Cd}$ - Cadmium - 112.414(4) & 112.4144 & 49.45202042 & 0.573574204 \\
\hline 49 In - Indium - 114.818(1) & 114.8181 & 48.41674966 & 0.561566513 \\
\hline $50 \mathrm{Sn}-\mathrm{Tin}-118.710(7)$ & 118.7107 & 46.82913338 & 0.543152386 \\
\hline $51 \mathrm{Sb}$ - Antimony - 121.760(1) & 121.7601 & 45.65632916 & 0.529549499 \\
\hline $52 \mathrm{Te}$ - Tellurium - 127.60(3) & 127.603 & 43.56574065 & 0.505301599 \\
\hline 53 I - Iodine - $126.90447(3)$ & 126.904473 & 43.80554186 & 0.508082958 \\
\hline $54 \mathrm{Xe}-\mathrm{Xenon}-131.293(6)$ & 131.2936 & 42.34112862 & 0.491097814 \\
\hline 55 Cs - Cesium - 132.905451 96(6) & 132.905452 & 41.82762349 & 0.485141874 \\
\hline $56 \mathrm{Ba}$ - Barium - 137.327(7) & 137.3277 & 40.48068382 & 0.469519259 \\
\hline $57 \mathrm{La}$ - Lanthanum - 138.905 47(7) & 138.905477 & 40.0208784 & 0.46418616 \\
\hline $58 \mathrm{Ce}-$ Cerium - 140.116(1) & 140.1161 & 39.67509233 & 0.460175526 \\
\hline 59 Pr - Praseodymium - 140.907 66(2) & 140.907662 & 39.45221378 & 0.457590447 \\
\hline $60 \mathrm{Nd}$ - Neodymium - 144.242(3) & 144.2423 & 38.54014533 & 0.44701173 \\
\hline $61 \mathrm{Pm}-$ Promethium - <145> & 145 & 38.33875313 & 0.444675862 \\
\hline $62 \mathrm{Sm}$ - Samarium - 150.36(2) & 150.362 & 36.97156997 & 0.428818451 \\
\hline $63 \mathrm{Eu}$ - Europium - 151.964(1) & 151.9641 & 36.5817927 & 0.424297581 \\
\hline 64 Gd - Gadolinium - 157.25(3) & 157.253 & 35.35143497 & 0.410027154 \\
\hline $65 \mathrm{~Tb}$ - Terbium - $158.92535(2)$ & 158.925352 & 34.97943616 & 0.405712488 \\
\hline 66 Dy - Dysprosium - 162.500(1) & 162.5001 & 34.20994328 & 0.396787448 \\
\hline 67 Ho - Holmium - 164.930 33(2) & 164.930332 & 33.70586318 & 0.390940825 \\
\hline 68 Er - Erbium - 167.259(3) & 167.2593 & 33.23653276 & 0.385497249 \\
\hline $69 \mathrm{Tm}$ - Thulium - 168.934 22(2) & 168.934222 & 32.9070045 & 0.381675182 \\
\hline $70 \mathrm{Yb}$ - Ytterbium - 173.054(5) & 173.0545 & 32.12351718 & 0.372587826 \\
\hline $71 \mathrm{Lu}$ - Lutetium - 174.9668(1) & 174.96681 & 31.77242132 & 0.368515606 \\
\hline 72 Hf - Hafnium - 178.49(2) & 178.492 & 31.1449208 & 0.361237478 \\
\hline $73 \mathrm{Ta}$ - Tantalum - 180.947 88(2) & 180.947882 & 30.72221207 & 0.356334649 \\
\hline $74 \mathrm{~W}$ - Tungsten - 183.84(1) & 183.841 & 30.23873458 & 0.350726987 \\
\hline 75 Re - Rhenium - 186.207(1) & 186.2071 & 29.85449644 & 0.346270362 \\
\hline 76 Os - Osmium - 190.23(3) & 190.233 & 29.22268589 & 0.338942245 \\
\hline 77 Ir - Iridium - 192.217(3) & 192.2173 & 28.92101389 & 0.335443272 \\
\hline 78 Pt - Platinum - 195.084(9) & 195.0849 & 28.49589694 & 0.33051251 \\
\hline $79 \mathrm{Au}$ - Gold - 196.966 569(5) & 196.9665695 & 28.22366871 & 0.327355044 \\
\hline
\end{tabular}




\section{Continued}

\begin{tabular}{|c|c|c|c|}
\hline $80 \mathrm{Hg}$ - Mercury - 200.592(3) & 200.5923 & 27.71352242 & 0.321438061 \\
\hline $81 \mathrm{Tl}$ - Thallium - [204.382; 204.385] & 204.382 & 27.19965165 & 0.31547788 \\
\hline $82 \mathrm{~Pb}$ - Lead - 207.2(1) & 207.21 & 26.82843108 & 0.311172241 \\
\hline $83 \mathrm{Bi}$ - Bismuth - $208.98040(1)$ & 208.980401 & 26.601151 & 0.30853611 \\
\hline 84 Po - Polonium - <209> & 209 & 26.59865648 & 0.308507177 \\
\hline 85 At - Astatine $-<210>$ & 210 & 26.47199621 & 0.307038095 \\
\hline $86 \mathrm{Rn}-$ Radon $-<222>$ & 222 & 25.0410775 & 0.290441441 \\
\hline $87 \mathrm{Fr}-$ Francium $-<223>$ & 223 & 24.92878567 & 0.289139013 \\
\hline $88 \mathrm{Ra}$ - Radium - <226> & 226 & 24.59787258 & 0.285300885 \\
\hline 89 Ac - Actinium - $<227>$ & 227 & 24.48951191 & 0.284044053 \\
\hline $90 \mathrm{Th}$ - Thorium - 232.037 7(4) & 232.03774 & 23.95782343 & 0.277877211 \\
\hline $91 \mathrm{~Pa}$ - Protactinium - 231.035 88(2) & 231.035882 & 24.06171351 & 0.27908219 \\
\hline 92 U - Uranium - 238.028 91(3) & 238.028913 & 23.3548065 & 0.270883059 \\
\hline $93 \mathrm{~Np}$ - Neptunium - <237> & 237 & 23.45619917 & 0.272059072 \\
\hline $94 \mathrm{Pu}-$ Plutonium - <244> & 244 & 22.78327543 & 0.264254098 \\
\hline 95 Am - Americium - <243> & 243 & 22.87703376 & 0.265341564 \\
\hline $96 \mathrm{Cm}-$ Curium $-<247>$ & 247 & 22.50655548 & 0.261044534 \\
\hline 97 Bk - Berkelium - <247> & 247 & 22.50655548 & 0.261044534 \\
\hline $98 \mathrm{Cf}-$ Californium - $<251>$ & 251 & 22.14788528 & 0.256884462 \\
\hline 99 Es - Einsteinium - <252> & 252 & 22.05999684 & 0.255865079 \\
\hline $100 \mathrm{Fm}-$ Fermium $-<257>$ & 257 & 21.63081402 & 0.25088716 \\
\hline $101 \mathrm{Md}$ - Mendelevium - <258> & 258 & 21.54697366 & 0.249914729 \\
\hline 102 No - Nobelium - <259> & 259 & 21.46378071 & 0.248949807 \\
\hline $103 \mathrm{Lr}-$ Lawrencium $-<262>$ & 262 & 21.21801223 & 0.246099237 \\
\hline 104 Rf - Rutherfordium - <267> & 267 & 20.82067118 & 0.241490637 \\
\hline $105 \mathrm{Db}-$ Dubnium $-<268>$ & 268 & 20.7429821 & 0.240589552 \\
\hline $106 \mathrm{Sg}-$ Seaborgium $-<271>$ & 271 & 20.513355 & 0.237926199 \\
\hline $107 \mathrm{Bh}-$ Bohrium $-<272>$ & 272 & 20.43793825 & 0.237051471 \\
\hline $108 \mathrm{Hs}$ - Hassium - <270> & 270 & 20.58933039 & 0.238807407 \\
\hline $109 \mathrm{Mt}$ - Meitnerium - <276> & 276 & 20.14173625 & 0.233615942 \\
\hline 110 Ds - Darmstadtium - <281> & 281 & 19.78334236 & 0.229459075 \\
\hline $111 \mathrm{Rg}$ - Roentgenium $-<280>$ & 285 & 19.50568142 & 0.226238596 \\
\hline 112 Cn - Copernicium - $<285>$ & 285 & 19.50568142 & 0.226238596 \\
\hline 113 Uut - Ununtrium $-<284>$ & 284 & 19.57436339 & 0.227035211 \\
\hline $114 \mathrm{Fl}$ - Flerovium - <289> & 289 & 19.23570659 & 0.223107266 \\
\hline 115 Uup - Ununpentium - <288> & 288 & 19.30249724 & 0.223881944 \\
\hline 116 Lv - Livermorium - <293> & 293 & 18.97310309 & 0.220061433 \\
\hline 117 Uus- $<294>$ & 294 & 18.90856872 & 0.219312925 \\
\hline \multirow[t]{2}{*}{118 Uuo - Ununoctium - <294> } & 294 & 18.90856872 & 0.219312925 \\
\hline & Average & 140.3485256 & 1.627846409 \\
\hline
\end{tabular}


Table 5. Comparison of QGP energy outcome with other energy sources.

\begin{tabular}{ccc}
\hline No. & Energy Source & Output Energy, MeV \\
\hline 1 & QGP of Hydrogen & $5515 /$ atomic unit \\
2 & QGP of Helium & $1389 /$ atomic unit \\
3 & QGP of Lead & $207 /$ atomic unit \\
4 & QGP of Gold & $197 /$ atomic unit \\
5 & Nuclear Fission & $0.8 /$ atomic unit \\
6 & Nuclear Fusion & $3.5 /$ atomic unit \\
7 & Hydrogen Ion annihilation & $938 /$ atomic unit \\
\hline
\end{tabular}

$926 \mathrm{MeV}$ gluon energy.

Moreover, this research has investigated the expansion of the universe based on the thermodynamics isothermal concept. It is concluded that the universe rate of change of pressure with respect of its volume is inversely proportional to the negative of squared volume $-1 / V^{2}$. Furthermore, since the dark energy pressure is so tiny, $8.8981 \times 10^{-10} \mathrm{~J} / \mathrm{m}^{3}$, but not zero, then it yields that the universe volume is not infinite and flat with current universe pressure to volume rate of change $-1.155 \times 10^{-88} \mathrm{~N} / \mathrm{m}^{2} / \mathrm{m}^{3}$.

Furthermore, the individual critical temperature of the quark-gluon plasma matter at which the elements of the Periodic Table are generated is determined. Based on the results, a novel Periodic Table is introduced. It can be seen that the highest critical temperature needed to generate quark-gluon plasma (QGP) from Hydrogen atom is 64 TK $(5516 \mathrm{MeV})$, followed by $16.1 \mathrm{TK}(1389 \mathrm{MeV})$ needed to generate QGP from Helium, and ended by the 0.219 TK $(18.9 \mathrm{MeV})$ to produce QGP from the last Periodic undiscovered yet element. It is interesting to know that the critical temperatures at which QGP is generated out of Gold atom and Uranium atom are $0.327 \mathrm{TK}(28.8 \mathrm{MeV})$ and $0.271(23.4 \mathrm{MeV})$, respectively. The average QGP critical temperature of all natural elements is figured to be $1.627 \mathrm{TK}(18.9 \mathrm{MeV})$.

Additionally, the QGP critical temperature trend of the elements of the Periodic Table Groups and Periods is then tested. It is figured out that the overall trend is of negative power regression $y=40.07 x^{-1.099}$ (TK) in Temperature scale and $y=3455.1 x^{-1.099}(\mathrm{MeV})$ in energy scale. This hints how is the trend at which the elements are created after the Big Bang. The QGP critical temperature is decreasing dramatically going down from Hydrogen, through Helium and ending by the Transition metals at Period 7. Then, the QGP critical temperatures of the Periods and the groups are examined separately. The critical temperature of Periods is found to have either linear trend with negative slope or quadratic decreasing trend. The first Period takes the linear form, followed by a quadratic form for the second Period, followed by a linear trend for the third Period, followed by quadratic form for the fourth Period, and then followed by a linear trend for all successive Periods fifth, sixth and the seventh. It is noticed that the negative slope is increasing by going down from the top Period to the bottom Period from slope -47.867 to -0.00038 . The quadratic coefficient is also decreasing from 0.1078 in Period 2 to 0.0019 in Period 4 .

On the other hand, the QGP critical temperatures of the groups are investigated. The critical temperature of groups is found to have negative power aggression trend. It is found out that both the power coefficient and negative exponent are decreasing as we go from left side of the groups A to the right side of groups A. Similarly, groups B are showing a decreasing QGP critical temperature pattern. It noticed that the negative slope is increasing by going down from the top Period to the bottom Period. For example, Group 1A shows a power trend of $y=63.055 x^{-2.737}$ (TK) (or $y=10.356 x^{-1.902}$ (TK) * excluding H), and ends with $y=16.077 x^{-2.199}$ (TK) (or $y=3.7873 x^{-1.532}$ (TK) Excluding He). Likely, Groups B (starting with 4B, 5B, 6B, 7B, 8B1, 8B2, 8B3, 1B and $2 \mathrm{~B}$ ) demonstrate negative power regression starting with $y=1.4459 x^{-1.247}$ and ending with $y=1.0516 x^{-1.067}$ (TK). The exception to this pattern is Group 3B which shows linearly decreasing trend beginning with $y=1.5012 x^{-1.136}$ (TK) and terminating with behavior $y=-0.1224 x+0.4909$ (TK). The QGP critical temperatures of lanthanide and actinide series pattern demonstrate a decreasing linear regression trend.

A new QGP phase diagram depicts that the Hardon Epoch is proposed in this work. The QGP phase diagram presents a critical point that separates the two phases: the hadron gas in which quarks are confined, and the quark-gluon plasma (QGP). Finally, a novel Quark-Gluon Plasma power generation reactor is proposed. It basi- 
cally utilizes the output temperatures to heat up cold water and converts the water into a steam. The pressurized steam will generate a high mechanical rotational at the turbines and then high induced electrical power at the electrical generators. It is remarkable to note here that the outcome energy of the QGP range [5516 to $18.9 \mathrm{MeV}$ ] will be much higher compared with the nuclear fission which generates $0.8 \mathrm{MeV}$ per atomic unit and $3.5 \mathrm{MeV}$ per atomic unit that can be produced from the nuclear fusion. Future work will elaborate more on this QCD power plant analysis and design.

\section{References}

[1] Knop, R.A., Aldering, G., Amanullah, R., Astier, P., Blanc, G., Burns, M.S., et al. (2003) New Constraints on $\Omega_{\mathrm{M}}, \Omega_{\Lambda}$, and $w$ from an Independent Set of Eleven High-Redshift Supernovae Observed with HST. Astrophysical Journal, 598, 102-137. http://dx.doi.org/10.1086/378560

[2] Permutter, S., Aldering, G., Goldhaber, G., Knop, R.A., Nugent, P., Castro, P.G., et al. (1999) Measurements of Omega and Lambda from 42 High Redshift Supernovae. Astrophysical Journal, 517, 565-586. http://dx.doi.org/10.1086/307221

[3] Riess, A.G., Filippenko, A.V., Challis, P., Clocchiatti, A., Diercks, A., Garnavich, P.M., et al. (1998) Observational Evidence from Supernovae for an Accelerating Universe and Cosmological Constant. Astronomical Journal, 116, 1009-1038. http://dx.doi.org/10.1086/300499

[4] Maartens, R. and Majerotto, E. (2006) Observational Constraints on Self-Accelerating Cosmology. Journal of Astrophysics, 74, 2-6. http://dx.doi.org/10.1103/physrevd.74.023004

[5] Perlmutter, S., Turner, M.S. and White, M. (1999) Constraining Dark Energy with SNe Ia and Large-Scale Structure. Physical Review Letters, 83, 670-673. http://dx.doi.org/10.1103/PhysRevLett.83.670

[6] Freedman, W.L., Madore, B.F., Gibson, B.K., Ferrarese, L., Kelson, D.D., Sakai, S., et al. (2000) Final Results from the Hubble Space Telescope Key Project to Measure the Hubble Constant. Journal of Astrophysics, 553, 47-72. http://dx.doi.org/10.1086/320638

[7] Tonry, J.L., Schmidt, B.P., Barris, B., Candia, P., Challis, P., Clocchiatti, A., et al. (2003) Cosmological Results from High-z Supernovae. Journal of Astrophysics, 594, 1-24. http://dx.doi.org/10.1086/376865

[8] Spergel, D.N., Bean, R., Doré, O., Nolta, M.R., Bennett, C.L., Dunkley, J., et al. (2006) Wilkinson Microwave Anisotropy Probe (WMAP) Three Year Results: Implications for Cosmology. WMAP Collaboration.

[9] Carroll, S.M. (2003) Why Is the Universe Accelerating? AIP Conference Proceedings, 743, 16-32. http://dx.doi.org/10.1063/1.1848314

[10] Einstein, A. (1914-1917) The Foundation of the General Theory of Relativity. [The Collected Papers of Einstein, English Translation Edited by Kox, A.J., Klien, M.J. and Schulmann, R., Vol. 6, Princeton University Press, 1997]. The Berlin Years Writing, 146-200.

[11] Shibli, M. (2007) The Foundation of the Fourth Law of Thermodynamics: Dark Energy and Its Nature: Can Dark Energy Be Generated? International Conference on Renewable Energies and Power Quality (ICREPQ'07), Seville, 26-28 March 2007, European Association for the Development of Renewable Energies, Environment and Power Quality. www.icrepq.com

[12] Turner, M.S. (1999) Dark Matter and Dark Energy in the Universe. The Third Astro. Symposium: The Galactic Halo ASP Conference Series, Vol. 666.

[13] Huterer, D. and Turner, M.S. (1999) Prospects for Probing the Dark Energy via Supernova Distance Measurements. Physical Review D, 60, Article ID: 081301. http://dx.doi.org/10.1103/physrevd.60.081301

[14] Carroll, S.M., Hoffman, M. and Trodden, M. (2003) Can the Dark Energy Equation-of-State Parameter $w$ Be Less than -1? Physical Review D, 68, Article ID: 023509. http://dx.doi.org/10.1103/PhysRevD.68.023509

[15] Huterer, D. and Turner, M.S. (2001) Probing Dark Energy: Methods and Strategies. Physical Review D, 64, Article ID: 123527. http://dx.doi.org/10.1103/physrevd.64.123527

[16] Peebles, P.J.E. and Ratra, B. (2003) The Cosmological Constant and Dark Energy. Reviews of Modern Physics, 75, 559-606. http://dx.doi.org/10.1103/revmodphys.75.559

[17] Copeland, E.J., Sami, M. and Tsujikawa, S. (2006) Dynamics of Dark Energy. International Journal of Modern Physics D, 15, 1753-1935. http://dx.doi.org/10.1142/S021827180600942X

[18] Volovik, G.E. (2006) Vcauum Energy: Myths and Reality. International Journal of Modern Physics D, 15, $1987-2010$. http://dx.doi.org/10.1142/S0218271806009431

[19] Carroll, S.M., Sawicki, I., Silvestri, A. and Trodden, M. (2006) Modified-Source Gravity and Cosmological Structure Formation. New Journal of Physics, 8, 323. http://dx.doi.org/10.1088/1367-2630/8/12/323 
[20] Carroll, S.M. (2001) The Cosmological Constant. Living Reviews of Relativity, 4. http://dx.doi.org/10.12942/lrr-2001-1

[21] Dvali, G. and Turner, M. (2003) Dark Energy as a Modifications of the Friedmann Equation. Journal of Astrophysics, 2,11 .

[22] Spergel, D.N., Bean, R., Doré, O., Nolta, M.R., Bennett, C.L., Dunkley, J., et al. (2007) Three-Year Wilkinson Microwave Anisotropy Probe (WMAP) Observations. The Astrophysical Journal Supplement Series, 170, 377-408. http://dx.doi.org/10.1086/513700

[23] Komatsu, E., Smith, K.M., Dunkley, J., et al. (2010) Seven-Year Wilkinson Microwave Anisotropy Probe (WMAP) Observations: Cosmological Interpretation. Astrophysical Journal Supplement Series. http://arxiv.org/abs/1001.4538v3

[24] Spergel, D.N., Verde, L., Peiris, H.V., Komatsu, E., Nolta, M.R., Bennett, C.L., et al. (2003) First Year Wilkinson Microwave Anisotropy Probe (WMAP) Observations: Determination of Cosmological Parameters. The Astrophysical Journal Supplement Series, 148, 175-194. http://dx.doi.org/10.1086/377226

[25] Shibli, M. (2011) The Foundation of the Theory of the Universe Dark Energy and Its Nature. Natural Science, 3, 165-185. http://dx.doi.org/10.4236/ns.2011.33023

[26] Jacak, B.V. and Müller, B. (2015) The Exploration of Hot Nuclear Matter. Science, 337, 310-314. http://dx.doi.org/10.1126/science.1215901

[27] US Department of Energy's (DOE) Brookhaven National Laboratory (2010) New Findings on Hot Quark Soup Produced at RHIC.

[28] Stephanov, M.A. (2006) QCD Phase Diagram: An Overview. High Energy Physics-Lattice, arXiv.org.

[29] Fukao, Y. (2006) The Overview of the Spin Physics at RHIC-PHENIX Experiment. High Energy Physics, arXiv.org.

[30] Sowinski, J. (2007) Exploring the Spin Structure of the Proton with Two-Body Partonic Scattering at RHIC. High Energy Physics, arXiv.org.

[31] Song, H.C., Bass, S.A., Heinz, U.W., Hirano, T. and Shen, C. (2011) $200 \mathrm{~A} \mathrm{GeV} \mathrm{Au+Au} \mathrm{Collisions} \mathrm{Serve} \mathrm{a} \mathrm{Nearly}$ Perfect Quark-Gluon Liquid. Physical Review Letters, 106, Article ID: 192301. http://dx.doi.org/10.1103/PhysRevLett.106.192301

[32] Cheng, M., Christ, N.H., Datta, S., van der Heide, J., Jung, C., Karsch, F., Kaczmarek, O., Laermann, E., Mawhinney, R.D., Miao, C., Petreczky, P., Petrov, K., Schmidt, C., Soeldner, W. and Umeda, T. (2008) The QCD Equation of State with Almost Physical Quark Masses. Physical Review D, 77, Article ID: 014511. http://dx.doi.org/10.1103/PhysRevD.77.014511

[33] Cheng, M., Christ, N.H., Datta, S., van der Heide, J., Jung, C., Karsch, F., Kaczmarek, O., Laermann, E., Mawhinney, R.D., Miao, C., Petreczky, P., Petrov, K., Schmidt, C. and Umeda, T. (2006) The Transition Temperature in QCD. Physical Review D, 74, Article ID: 054507. http://dx.doi.org/10.1103/PhysRevD.74.054507

[34] Cengel, Y.A. and Boles, M.A. (2006) Thermodynamics: An Engineering Approach. Fifth Edition, McGraw Hill, New York.

[35] Coughlan, G.D., Dodd, J.E. and Gripaios, B.M. (2006) The Ideas of Particle Physics: An Introduction for Scientists.

[36] Hawking, S.W. (1998) A Brief History of Time: From the Big Bang to Black Holes. Bantam Books, Inc., New York.

[37] Shibli, M. (2008) The Equation of State of Dark Energy and Dark Matter: Boltzmann Constant and the Unified Entity: Utilization of Space Energy. ICREPQ'08, Santander, 12-14 March 2008, European Association for the Development of Renewable Energies, Environment and Power Quality.

[38] Shibli, M. (2009) The Fundamental Particle and Energy Quanta of Dark Matter and Dark Energy: Boltzmann Particles and Utilization Its Energy. International Conference on Renewable Energies and Power Quality (ICREPQ'09), Valencia, 15-17 April 2009, pp.

[39] Freese, K. and Kinney, W.H. (2002) The Ultimate Fate of Life in an Accelerating Universe. Physics Letters B, 588, 1-8. http://dx.doi.org/10.1016/S0370-2693(02)02122-6

[40] Mohr, P.J., Taylor, B.N. and Newell, D.B. (2007) CODATA Recommended Values of the Fundamental Physical Constants. National Institute of Standards and Technology, Gaithersburg.

[41] Emsley, J. (2001) Nature's Building Blocks: An A-Z Guide to the Elements (New Edition). Oxford University Press, New York.

[42] Shibli, M. (2011) The Basic Blocks of the Universe Matter: Boltzmann Fundamental Particle and Energy Quanta of Dark Matter and Dark Energy. Natural Science, 3, 743-749. http://dx.doi.org/10.4236/ns.2011.39099 